\title{
TRANSCENDENCE MEASURES AND ALGEBRAIC GROWTH OF ENTIRE FUNCTIONS
}

\author{
DAN COMAN AND EVGENY A. POLETSKY
}

\begin{abstract}
In this paper we obtain estimates for certain transcendence measures of an entire function $f$. Using these estimates, we prove Bernstein, doubling and Markov inequalities for a polynomial $P(z, w)$ in $\mathbb{C}^{2}$ along the graph of $f$. These inequalities provide, in turn, estimates for the number of zeros of the function $P(z, f(z))$ in the disk of radius $r$, in terms of the degree of $P$ and of $r$.

Our estimates hold for arbitrary entire functions $f$ of finite order, and for a subsequence $\left\{n_{j}\right\}$ of degrees of polynomials. But for special classes of functions, including the Riemann $\zeta$-function, they hold for all degrees and are asymptotically best possible. From this theory we derive lower estimates for a certain algebraic measure of a set of values $f(E)$, in terms of the size of the set $E$.
\end{abstract}

\section{INTRODUCTION}

In recent years there was a significant interest in the behavior of a polynomial $P$ along an algebraic subvariety $X$ of $\mathbb{R}^{n}$ or $\mathbb{C}^{n}$. This started with the paper [FN] of Fefferman and Narasimhan, where they obtained local doubling inequalities, which bound the ratio of the uniform norms of $P$ on two concentric balls in $X$, in terms of the degrees of $P$ and $X$, and of the ratio of the radii of these balls.

Later, these inequalities were improved in papers of Brudnyi $[\mathrm{Br}]$ and Roytwarf and Yomdin [RY], and they were applied to questions from analytic geometry, pseudodifferential operators, to Hilbert's 16th problem, and so on.

Much earlier, Tijdeman [Ti1] studied the behavior of a polynomial $P(z, w)$ in $\mathbb{C}^{2}$ along the graph of the exponential function $w=e^{z}$. In this situation, he obtained global doubling inequalities and estimates for the number of zeros of the function $P\left(z, e^{z}\right)$ in a disk of radius $r$. He used these results in [Ti2] to get new advancements in transcendental number theory. The proofs in [Ti2] involved transcendence measures

Date: March 2004.

2000 Mathematics Subject Classification. Primary: 30D15; Secondary 11J99, 30D20.

Both authors are supported by NSF Grants. 
of numbers, which were studied extensively in transcendental number theory.

Transcendence measures appear quite naturally when transcendental objects are investigated. In general, if $B$ is a subring of a commutative $\operatorname{ring} A$, then an element $\omega \in A$ is called transcendental over $B$ if $P(\omega) \neq$ 0 , for any non-trivial polynomial $P \in B[x]$. For example, if $A=\mathbb{C}$ and $B=\mathbb{Z}$ we get the transcendental numbers, and if $A$ is the ring of entire functions and $\mathbb{C}[z]$ the ring of polynomials in $\mathbb{C}$, we get the entire transcendental functions.

If $A$ and $B$ are normed rings and the algebra $B[x]$ is graded, i.e., there is an increasing sequence of sets $B_{n}[x]$ such that $\bigcup_{n>0} B_{n}[x]=B[x]$, then we can measure the transcendence of $\omega$. For this, we define a suitable norm $h(P)$ of $P \in B[x]$, and let the transcendence measure $\tau(\omega, n, H)$ of $\omega$ be the infimum of $\|P(\omega)\|$ over all polynomials $P \in$ $B_{n}[x]$ with $1 \leq h(P) \leq H$.

In our papers [CP1] and [CP2], we started to study transcendence measures of an entire function $f$. A transcendence measure can be defined by

$$
E_{n}(f)=\sup \left\{\|P\|_{\Delta^{2}}: P \in \mathbb{C}[z, w], \operatorname{deg} P \leq n,\|P(z, f(z))\|_{\Delta} \leq 1\right\} .
$$

Here $\Delta$ is the closed unit disk in $\mathbb{C}$ and the norms are uniform norms. Since $f$ is usually fixed, we write $E_{n}=E_{n}(f)$, and let $e_{n}=\log E_{n}$. In $[\mathrm{CP} 1]$ we proved that

$$
e_{n}\left(e^{z}\right)=\frac{1}{2} n^{2} \log n+O\left(n^{2}\right) .
$$

This transcendence measure is closely connected with the following aspects of analysis and geometry:

1) Polynomial estimates on $\mathbb{C}^{2}$ : if $P(z, w)$ is a polynomial of degree $n$ and $|P(z, f(z))| \leq 1$ on the unit disk $\Delta$, then

$$
|P(z, w)| \leq E_{n} \exp \left(n \max \left\{\log ^{+}|z|, \log ^{+}|w|\right\}\right) ;
$$

2) Polynomial estimates along the graph of $f$ : if

$$
\begin{gathered}
m(r)=m(r, f)=\max \left\{\log ^{+}|f(z)|:|z|=r\right\}, \\
P_{f}(z)=P(z, f(z)), \Delta_{r}=\{z \in \mathbb{C}:|z| \leq r\} \text { and } \\
m_{n}(r)=m_{n}(r, f)=\sup \left\{\log \left\|P_{f}\right\|_{\Delta_{r}}: \operatorname{deg} P \leq n,\left\|P_{f}\right\|_{\Delta} \leq 1\right\},
\end{gathered}
$$

then

$$
m_{n}(r) \leq \frac{2 e_{n}}{\log t_{n}} \log r, 1 \leq r \leq t_{n}
$$


where $t_{n}$ is defined by $n m\left(t_{n}\right)=e_{n}$. The functions $m_{n}(r, f), r>1$, can also be considered as transcendence measures of $f$, by using $\left\|P_{f}\right\|_{\Delta_{r}}$ as the norm of a polynomial $P(z, w)$.

3) Estimates on the number of zeros: if $Z_{n}(r)=Z_{n}(r, f)$ is the maximum number of zeros of the function $P_{f}$ in the disk $\Delta_{r}$ when $\operatorname{deg} P \leq n$, then $Z_{n}(r) \leq 2 m_{n}(3 r)$. The number $Z_{n}(r)$ gives the maximum number of intersection points of an algebraic variety of degree $n$ with the graph of $f$ in $\mathbb{C}^{2}$ lying over $\Delta_{r}$.

These connections were proved in [CP2], where we also found an approach to estimate $e_{n}$ for general transcendental functions. It allowed us to handle, in particular, the class of functions $f(z)=e^{P(z)}$, where $P$ is a polynomial.

For any transcendental function $f$ one has [CP1, Proposition 1.3]

$$
m_{n}(r, f) \geq \frac{n^{2}+3 n}{2} \log r, r \geq 1 .
$$

Using the transcendence measure $m_{n}(e, f)$, we define the lower order of transcendence as

$$
\underline{\tau}(f)=\sup \left\{\tau: \liminf _{n \rightarrow \infty} \frac{m_{n}(e, f)}{n^{\tau}}>0\right\},
$$

and the upper order of transcendence as

$$
\bar{\tau}(f)=\inf \left\{\tau: \limsup _{n \rightarrow \infty} \frac{m_{n}(e, f)}{n^{\tau}}<\infty\right\} .
$$

Since $m_{n}(e, f) \geq n^{2} / 2$, we have $\underline{\tau}(f) \geq 2$. If $f(z)=e^{z}$ then $\lim _{n \rightarrow \infty} m_{n}(e) / n^{2}=1 / 2$ [CP1, Theorem 1.2]. More generally, if $f(z)=$ $e^{P(z)}$, for some polynomial $P$, then $\underline{\tau}(f)=\bar{\tau}(f)=2[\mathrm{CP} 2$, Theorem 5.1]. For $\tau \geq 3$, we constructed examples of entire functions of order 1 and type $1 / e$ with $\tau-1 \leq \bar{\tau}(f) \leq \tau$ [CP2, Corollary 6.2]. In all these examples, $\underline{\tau}(f)=2$. Whether this was true in general remained unsettled until the present paper.

The approach in [CP2] was based on estimates of $e_{n}(f)$ in terms of the $n$-th diameter of the set of preimages of a point on the unit circle. The $n$-th diameter $d_{n}(F)$ of a set $F$ is the minimal sum of radii of $n$ disks covering $F$. In [So], using the theory of Dufresnoy, Sodin gave lower bounds for the smallest number of disks of radius $R^{\alpha}$, $\alpha<1$, needed to cover the set $f^{-1}(\{0,1\}) \cap \Delta_{R}$, when $f$ is a function of finite positive order $\rho$. Applied to our problem, his result leads to only exponential estimates for $e_{n}$. In Section 3, using the Ahlfors theory of covering surfaces and certain results of Dufresnoy, we obtain the necessary estimates for the $n$-th diameter. The results we need from these theories are recalled in Section 2. 
The estimates for the $n$-th diameter allow us to obtain several results, which can be summarized in the following theorem. In this theorem, the second inequality is usually called a Bernstein inequality, the third - a Bezout inequality, the fourth - a doubling inequality, and the fifth - a Markov inequality. Bernstein and Markov inequalities have been extensively studied and have wide applications, for example in approximation theory (see e.g. [BBLT] and references therein).

Theorem 1.1. For any entire function $f$ of finite order $\rho>0$, there exist sequences of integers $\left\{n_{j}\right\}$ and $\epsilon_{j}>0, \epsilon_{j} \rightarrow 0$, such that

$$
e_{n_{j}} \leq C_{1} n_{j}^{2} \log n_{j}, m_{n_{j}}(r) \leq C_{2} n_{j}^{2} \log r, 1 \leq r \leq \frac{1}{2} n_{j}^{1 / \rho-\epsilon_{j}} .
$$

For every $r \geq 1$ there exists an integer $j_{r}$ such that if $j \geq j_{r}$ then

$$
Z_{n_{j}}(r) \leq C_{3} n_{j}^{2}, \frac{M\left(2 r, P_{f}\right)}{M\left(r, P_{f}\right)} \leq 2^{a n_{j}^{2}}, \quad M\left(r, P_{f}^{\prime}\right) \leq C_{4} n_{j}^{2} \frac{M\left(r, P_{f}\right)}{r},
$$

where $P$ is a polynomial of degree at most $n_{j}$.

Here $M(r, F)=\max \{|F(z)|:|z|=r\}$ and the constants are effectively computed and depend only on $\rho$. A sequence of integers $\left\{n_{j}\right\}$ for which the above theorem holds will be called a fundamental sequence for $f$. It follows from this theorem that $\underline{\tau}(f)=2$ for all entire functions of finite positive order.

Theorem 1.1 is proved in Sections 4, 5 and 6, where we also show that for entire functions with a covering system of admissible intervals $I(R, \alpha, \beta, \gamma, C)$ (see Section 4), the inequalities in Theorem 1.1 hold for all $n$ sufficiently large. Again, all constants are effectively computed. The only change is that one should substitute $n^{1+1 / \gamma}$ instead of $n_{j}^{2}$.

In Section 7 we give three sufficient criteria for classes of functions to have a covering system of admissible intervals $I(R, \alpha, \beta, \gamma, C)$. The first one states that if $A_{1} m(r, f) \leq m(k r, f) \leq A_{2} m(r, f)$, for some constants $A_{1}, A_{2}, k>1$, then the function $f$ has a covering system of admissible intervals $I(R, \alpha, \beta, 1, C)$. This class includes all functions $f(z)=\sum_{j=1}^{m} p_{j}(z) e^{q_{j}(z)}$, where $p_{j}$ and $q_{j}$ are polynomials, and, as shown in Section 8, the Riemann $\zeta$-function and the function $\xi$. It follows that for such functions Theorem 1.1 holds for all $n$ sufficiently large.

The second criterion can be applied when we know that $m(r, f) \leq$ $r^{\phi(r)}$ and $r^{\phi(r)-\rho}$ is a slowly increasing function (see Theorem 7.3). Finally, Corollary 7.4 gives a criterion based on the behavior of the Taylor coefficients of $f$, similar to the formulas for the order and type of $f$.

In Section 9 we introduce and study an extremal function $W^{\star}(z)$, related to Bernstein inequalities, and we prove that $W^{\star}(z)=\frac{1}{2} \log ^{+}|z|$ when $f(z)=e^{z}$. 
In Section 11 we address a problem posed by Mahler in [M]: given an entire transcendental function $f$, describe, or at least find properties of, the set of algebraic numbers where the values of $f$ are also algebraic. There are many results claiming that this set is finite when either $f$ is a special function, or when all the derivatives of $f$ take algebraic values on this set and their algebraic measure satisfies some growth conditions (see, e.g., [Sc], [St], [La], [W]). But a general entire function may take algebraic values on any set of algebraic numbers (see $[\mathrm{M}]$ and [GS]), in particular, on any algebraic number field $K$ of degree $\sigma$. So it is interesting to look at the algebraic growth characteristic $\mathbf{a}_{K}(s, r, m)$ of $f$, defined as the smallest algebraic measure of the first $m$ derivatives of $f$ on sets $E \subset \Delta_{r} \cap K$ with $|E| \geq s$ (see Section 11). The following theorem, proved in Section 11, gives lower bounds for this characteristic.

Theorem 1.2. If $f$ is an entire function of finite positive order then

$$
\limsup _{s \rightarrow \infty} \frac{\mathbf{a}_{K}(s, r, m)}{s^{1 / 2} \log s} \geq C m^{1 / 2} .
$$

If $f$ has a covering system of admissible intervals $I(R, \alpha, \beta, \gamma, C)$, then for all s sufficiently large

$$
\mathbf{a}_{K}(s, r, m) \geq c(m s)^{1 / \tau} \log \frac{m s}{a}-C_{K}, \tau=1+\frac{1}{\gamma} .
$$

Let $I_{K}(A)$ be the set of algebraic integers in $K$ whose algebraic measure does not exceed $A$. Then it is possible that $f\left(I_{K}(A)\right)$ lies in some $I_{K}(B)$, like in the theorems of Polya and Gelfond (see [GS]), where $K=\mathbb{Q}$ or $\mathbb{Q}[i]$. Of course, $B$ can be large, simply due to the growth of $f$. However, if $m(r, f) \leq r^{\phi(r)}$ we prove in Section 11 the following theorem:

Theorem 1.3. If $f$ is an entire function of order $0<\rho<\sigma / 2$ then

$$
\liminf _{A \rightarrow \infty} \frac{\left|I_{K}(A) \cap f^{-1}\left(I_{K}\left(\exp A^{\phi(A)}\right)\right)\right|}{\left|I_{K}(A)\right|}=0 .
$$

This theorem tells us that, with probability close to 1 , the algebraic measure of $f(z)$ for $z \in I_{K}(A)$ growth faster than $f$.

To prove these and other theorems, we combine our Bezout inequalities with the standard machinery based on Siegel's lemma. This is developed in Section 10 and gives lower bounds for the algebraic measure of arguments and values of $f$ on a set $E \subset K$.

We are grateful to A. Eremenko and N. Levenberg for useful discussions. A. Eremenko also told us about Sodin's paper [So]. 


\section{Characteristics of EntiRe FunCtions}

For an entire function $f$ on $\mathbb{C}$ we let

$$
u_{f}(z)=\frac{1}{2} \log \left(1+|f|^{2}\right)
$$

This is a subharmonic function with Laplacian

$$
\Delta u_{f}(z)=2 \rho_{f}^{2}=\frac{2\left|f^{\prime}\right|^{2}}{\left(1+|f|^{2}\right)^{2}},
$$

where $\rho_{f}$ is the absolute value of the spherical derivative of $f$.

For a domain $D \subset \mathbb{C}$ with piecewise analytic boundary let

$$
\begin{aligned}
& L(D)=L_{f}(D)=2 \int_{\partial D} \rho_{f}|d z|, \\
& S(D)=S_{f}(D)=\frac{1}{\pi} \int_{D} \rho_{f}^{2} d \lambda=\frac{1}{2 \pi} \int_{D} \Delta u_{f},
\end{aligned}
$$

where $\lambda$ is the Lebesgue measure on $\mathbb{C}$. If $D=\Delta_{r}$ then

$$
L(D)=L(r)=2 \int_{|z|=r} \rho_{f}|d z|, S(D)=S(r)=\frac{1}{\pi} \int_{|z| \leq r} \rho_{f}^{2} d \lambda
$$

The following result of Ahlfors, with improvements by Dufresnoy, can be found in Chapters 5 and 6 of $[H]$ and [D, Theorem $A_{1}$, p. 190]:

Theorem 2.1. Let $f$ be an entire holomorphic function and let $D$ be a domain in $\mathbb{C}$ with piecewise analytic boundary and with Euler-Poincaré characteristic $\chi$. If $f$ does not assume in $D$ the values $a \neq b$, where $|a|=|b|=1$, then

$$
S(D) \leq \chi+1+\frac{3}{2 \delta_{0}} L(D)
$$

where $\delta_{0}$ is the spherical distance between a and $b$. Moreover, if $z \in D$ and $\operatorname{dist}(z, \partial D)=r$, then

$$
\rho_{f}(z) \leq \frac{e^{36 \pi^{2} / \delta_{0}^{2}}}{r}
$$

Here the Euler characteristic equals -2 for the sphere, -1 for the disk, and $\chi \geq 0$ for multiply connected domains.

The function

$$
T_{0}(r)=T_{0}(r, f)=\int_{0}^{r} \frac{S(t)}{t} d t
$$

is called the Ahlfors-Shimizu characteristic of $f$. If

$$
m_{0}(r)=m_{0}(r, f)=\frac{1}{2 \pi} \int_{0}^{2 \pi} \log \sqrt{1+\left|f\left(r e^{i \theta}\right)\right|^{2}} d \theta
$$


then (see $[H, \S 1.5])$

$$
T_{0}(r)=m_{0}(r)-\log \sqrt{1+|f(0)|^{2}} .
$$

We let

$$
\begin{aligned}
& M(r)=M(r, f)=\max \{|f(z)|:|z|=r\} \\
& m(r)=m(r, f)=\log ^{+} M(r, f) \\
& T(r)=T(r, f)=\frac{1}{2 \pi} \int_{0}^{2 \pi} \log ^{+}\left|f\left(r e^{i \theta}\right)\right| d \theta .
\end{aligned}
$$

By [H, Theorem 1.6] and [H, p. 13] we have

$$
\begin{aligned}
& T(r) \leq m(r) \leq \frac{R+r}{R-r} T(R), 0 \leq r<R, \\
& \left|T(r)-T_{0}(r)-\log ^{+}\right| f(0)|| \leq \frac{\log 2}{2} .
\end{aligned}
$$

The following relations between $L(r), S(r)$ and $T_{0}(r)$ will be important in the sequel. If $k>1$ then

$$
T_{0}(k r) \geq \int_{r}^{k r} \frac{S(t)}{t} d t \geq S(r) \log k .
$$

Moreover, Hölder's inequality implies for all $r$

$$
L^{2}(r) \leq 8 \pi^{2} r S^{\prime}(r)
$$

Note that if the function $S(r)$ is bounded then $f$ is a polynomial. Hence if $f$ is transcendental, $\epsilon>0, k>1$, we can define $r_{0}=r_{0}(f, \epsilon, k)$ by

$$
S\left(r_{0}\right)=\frac{8 \pi^{2}}{\epsilon^{2} \log k} .
$$

Lemma 2.2. If $r \geq r_{0}$ then there exists $r^{\prime} \in(r, k r)$ so that $L\left(r^{\prime}\right) \leq$ $\epsilon S\left(r^{\prime}\right)$.

Proof. Assuming that $L(t)>\epsilon S(t)$ for $t \in(r, k r)$, we have by (4) $\epsilon^{2} S^{2}(t)<8 \pi^{2} t S^{\prime}(t)$. Hence

$$
\frac{\epsilon^{2} \log k}{8 \pi^{2}}=\frac{\epsilon^{2}}{8 \pi^{2}} \int_{r}^{k r} \frac{d t}{t}<\int_{r}^{k r} \frac{S^{\prime}(t)}{S^{2}(t)} \leq \frac{1}{S\left(r_{0}\right)},
$$

a contradiction.

We will need the following facts about functions of finite order. Recall that (see [Le, Th.I.16]) if $\theta(r), r>0$, is a positive function with

$$
\rho=\limsup _{r \rightarrow \infty} \frac{\log \theta(r)}{\log r}<\infty
$$


then $\theta$ has a proximate order $\rho(r)$ with the following properties:

(i) $\lim _{r \rightarrow \infty} \rho(r)=\rho$;

(ii) $\theta(r) \leq r^{\rho(r)}$, and $\theta\left(r_{n}\right)=r_{n}^{\rho\left(r_{n}\right)}$ for some sequence $r_{n} \rightarrow \infty$;

(iii) the function $\psi(r)=r^{\rho(r)-\rho}$ is slowly increasing, i.e.,

$$
\lim _{r \rightarrow \infty} \frac{\psi(k r)}{\psi(r)}=1
$$

uniformly on each interval $0<a \leq k \leq b<\infty$. If $r^{\rho(r)-\rho}$ is a slowly increasing function, then for every $\epsilon>0$ and every $0<a<b<\infty$ there is $r_{0}$ such that

$$
(1-\epsilon) k^{\rho} r^{\rho(r)}<(k r)^{\rho(k r)}<(1+\epsilon) k^{\rho} r^{\rho(r)},
$$

for $a \leq k \leq b$ and $r \geq r_{0}$.

\section{ESTIMATES FOR THE $n$-TH DIAMETER}

We will need the following lemma:

Lemma 3.1. Let $u$ be a non-negative upper bounded subharmonic function in the disk $\Delta_{R}$. If $R^{\prime}=R / 2$ and $\Gamma=\Delta_{R^{\prime}} \cap \Delta(a, r)$, where $r<3 R / 4$, then

$$
\frac{1}{2 \pi} \int_{\Gamma} \Delta u \leq \frac{m_{0}}{\log \frac{3 R}{4 r}}
$$

where $m_{0}=\sup \{u(z):|z|<R\}$.

Proof. If $\Gamma \neq \emptyset$, there exists $b \in \Delta_{R^{\prime}}$ such that $\Gamma \subset \Delta(b, r)$. Since

$$
0 \leq u(b) \leq m_{0}+\frac{1}{2 \pi} \int_{\Delta_{R}} \log \left|\frac{R(z-b)}{R^{2}-\bar{b} z}\right| \Delta u(z)
$$

and

for $z \in \Gamma$, we obtain

$$
\left|\frac{R(z-b)}{R^{2}-\bar{b} z}\right| \leq \frac{r R}{R^{2}-R^{\prime 2}}=\frac{4 r}{3 R}
$$

$$
-m_{0} \leq \frac{\log \frac{4 r}{3 R}}{2 \pi} \int_{\Gamma} \Delta u(z)
$$

We introduce the constants

$$
\Lambda\left(\delta_{0}\right)=\left(4+\frac{48 \pi e^{36 \pi^{2} / \delta_{0}^{2}}}{\delta_{0}}\right)^{-1}, \Lambda=\Lambda(1) .
$$

The following theorem shows that the set of preimages of two points cannot be covered by a limited number of disks of small radius. 
Theorem 3.2. Let $f$ be an entire transcendental function and let $a, b \in$ $\mathbb{C},|a|=|b|=1$, and $\delta_{0}$ be the spherical distance between $a$ and $b$. If $L(R) \leq \delta_{0} S(R) / 6$ and if the set

$$
E=\left\{z \in \mathbb{C}:|z| \leq R+r, z \in f^{-1}(\{a, b\})\right\}
$$

is covered by $n \leq \Lambda\left(\delta_{0}\right) S(R)$ disks of radius $r$, then

$$
\log \frac{3 R}{4 r} \leq 4 n \frac{m(2 R)}{S(R)} .
$$

Proof. We assume at first that $3 n r>2 e^{-2} R$, so

$$
\log \frac{3 R}{4 r}<2+\log \frac{9}{8}+\log n .
$$

Using (3) and (2) we get

$$
S(R) \leq \frac{T_{0}(2 R)}{\log 2} \leq \frac{m(2 R)}{\log 2}+\frac{1}{2},
$$

so

$$
4 n \frac{m(2 R)}{S(R)} \geq 4 n \log 2\left(1-\frac{1}{2 S(R)}\right) .
$$

Since $n \leq \Lambda\left(\delta_{0}\right) S(R) \leq S(R) / 4$, we obtain

$$
4 n \frac{m(2 R)}{S(R)} \geq 4 n \log 2\left(1-\frac{1}{8 n}\right) \geq 2+\log \frac{9}{8}+\log n,
$$

for all $n \geq 1$. This proves Theorem 3.2 in the case $3 n r>2 e^{-2} R$.

We assume in the remainder of the proof that $3 n r \leq 2 e^{-2} R$. Suppose that the set $E$ can be covered by $n$ disks $\Delta\left(a_{j}, r\right)$ such that $n r=d$. We claim that there are disjoint disks $\Delta\left(b_{j}, t_{j}\right), 1 \leq j \leq k \leq n$, whose union contains all disks $\Delta\left(a_{j}, 2 r\right)$ and so that $\sum t_{j} \leq 2 d$. For this, we note that if the disks $\Delta\left(a_{1}, 2 r_{1}\right)$ and $\Delta\left(a_{2}, 2 r_{2}\right)$ are not disjoint, then there is a point $z$ such that the disk $\Delta\left(z, 2\left(r_{1}+r_{2}\right)\right)$ contains both these disks. Now a simple induction proves our claim.

We consider those disks $F_{j}=\Delta\left(b_{j}, t_{j}\right), j=1, \ldots, l$ which intersect $\Delta_{R}$. Let $\Gamma_{j}=F_{j} \cap \Delta_{R}$ and let $D=\Delta_{R} \backslash \cup_{j=1}^{l} F_{j}$. It follows that

$$
\Delta_{R}=D \cup \bigcup_{j=1}^{l} \Gamma_{j},
$$

thus

$$
S(R)=S(D)+\sum_{j=1}^{l} S\left(\Gamma_{j}\right)
$$


By (6) and the assumption that $S(R) \geq \Lambda^{-1}\left(\delta_{0}\right)$, it follows that $m(2 R) \geq \sqrt{2}$. Since $\log \left(1+x^{2}\right) \leq 4 \log x$ when $x \geq \sqrt{2}$, we get by Lemma 3.1 (with $u=\log \sqrt{1+|f|^{2}}$ )

$$
S\left(\Gamma_{j}\right) \leq 2 \frac{m(2 R)}{\log \frac{3 R}{2 t_{j}}} .
$$

Hence

$$
\sum_{j=1}^{l} S\left(\Gamma_{j}\right) \leq 2 m(2 R) \sum_{j=1}^{l} \frac{1}{\log \frac{3 R}{2 t_{j}}} .
$$

Since the sum of $2 t_{j} /(3 R)$ does not exceed $4 d /(3 R) \leq e^{-2}$ and the function $-1 / \log x$ is concave on $\left(0, e^{-2}\right)$ we conclude that

$$
\sum_{j=1}^{l} \frac{1}{\log \frac{3 R}{2 t_{j}}} \leq \frac{l}{\log \frac{3 R l}{4 d}} .
$$

As the function $x / \log a x$ is increasing when $x>e / a$ we have

$$
\frac{l}{\log \frac{3 R l}{4 d}} \leq \frac{n}{\log \frac{3 R}{4 r}} .
$$

Thus

$$
\sum_{j=1}^{l} S\left(\Gamma_{j}\right) \leq \frac{2 n m(2 R)}{\log \frac{3 R}{4 r}}
$$

Note that the Euler characteristic $\chi_{0}$ of $D$ verifies $\chi_{0} \leq n-1$, since the domain $D$ is bounded by at most $n+1$ Jordan curves. Moreover, we have

$$
L(\partial D) \leq L(R)+\sum_{j=1}^{l} L\left(\gamma_{j}\right)
$$

where $\gamma_{j}=\Delta_{R} \cap \partial \Delta\left(b_{j}, t_{j}\right)$. Thus Theorem 2.1 implies that

$$
S(D) \leq n+h L(R)+h \sum_{j=1}^{l} L\left(\gamma_{j}\right)
$$

where $h=3 /\left(2 \delta_{0}\right)$. If $z \in \gamma_{j}$, then $f$ does not take the values $a$ and $b$ in the disc $\Delta(z, r)$, so by Theorem $2.1 \rho_{f}(z) \leq h_{1} / r$, where $h_{1}=e^{36 \pi^{2} / \delta_{0}^{2}}$. Hence $L\left(\gamma_{j}\right) \leq 4 \pi h_{1} t_{j} / r$ and

$$
\sum_{j=1}^{l} L\left(\gamma_{j}\right) \leq \frac{8 \pi h_{1} d}{r}=8 \pi h_{1} n .
$$


We conclude that $S(D) \leq\left(1+8 \pi h h_{1}\right) n+h L(R)$, so

$$
S(R) \leq\left(1+8 \pi h h_{1}\right) n+h L(R)+\frac{2 n m(2 R)}{\log \frac{3 R}{4 r}} .
$$

If $n \leq \Lambda\left(\delta_{0}\right) S(R)$ and $L(R) \leq \delta_{0} S(R) / 6$, then

$$
\log \frac{3 R}{4 r} \leq \frac{4 n m(2 R)}{S(R)}
$$

For a set $G \subset \mathbb{C}$ and an integer $n \geq 1$ we introduced in [CP2] the $n$-th diameter of $G$ as

$$
\operatorname{diam}_{n}(G)=\inf \left\{r_{1}+\cdots+r_{k}: k \leq n, G \subset \bigcup_{j=1}^{k} C_{j}\left(r_{j}\right)\right\},
$$

where $C_{j}\left(r_{j}\right)$ are closed disks of radii $r_{j}>0$.

Given a non-constant entire function $f$ we denote in the sequel by $n_{0}=n_{0}(f)$ the maximum of the numbers $\left|f^{-1}(w) \cap \Delta_{2}\right|$ when $|w|=1$.

Corollary 3.3. In the assumptions of Theorem 3.2, let

$$
F=\left\{z \in \mathbb{C}: 2 \leq|z| \leq R+1, z \in f^{-1}(\{a, b\})\right\} .
$$

If $L(R) \leq \delta_{0} S(R) / 6, n \leq \Lambda\left(\delta_{0}\right) S(R)-2 n_{0}$, and $d_{n}=\operatorname{diam}_{n}(F)<1$, then

$$
\log \frac{R}{d_{n}} \leq 4\left(n+2 n_{0}\right) \frac{m(2 R)}{S(R)}+\log \frac{4}{3} .
$$

Proof. If $\epsilon>0$ and $d_{n}+\epsilon<1$, we can cover $F$ by $n$ disks of radius $d_{n}+\epsilon$. The number of points of $f^{-1}(\{a, b\}) \cap \Delta_{2}$ does not exceed $2 n_{0}$. We cover them with $2 n_{0}$ disks of radius $d_{n}+\epsilon$. Since $d_{n}+\epsilon<1$, we apply Theorem 3.2 and then let $\epsilon \rightarrow 0$.

Let

$$
D_{n}(\theta, r)=\left\{z \in \mathbb{C}: 2 \leq|z| \leq r, f(z)=e^{i \theta}\right\}
$$

and

$$
d_{n}(\theta, r)=\min \left\{1, \operatorname{diam}_{n}\left(D_{n}(\theta, r)\right)\right\} .
$$

Corollary 3.4. Let $f$ be an entire transcendental function. If $L(R) \leq$ $S(R) / 6$ and $n \leq \frac{1}{2} \Lambda S(R)-n_{0}$, then

$$
\log \frac{R}{d_{n}(\theta, R+1)} \leq \max \left\{8\left(n+n_{0}\right) \frac{m(2 R)}{S(R)}+\log 3, \log (2 R)\right\}
$$

for all $e^{i \theta}$ in an arc of length $l>\pi$ in $\partial \Delta$. 
Proof. Suppose that the spherical distance between $a=e^{i \phi}$ and $b=e^{i \psi}$ is at least $\delta_{0}=1$. Let $m=2 n$. If $F$ is as in Corollary 3.3, then

$$
\operatorname{diam}_{m}(F) \leq d_{n}(\phi, R+1)+d_{n}(\psi, R+1) .
$$

Hence if $\operatorname{diam}_{m}(F)<1$ we have by Corollary 3.3

$$
-\log \left(\frac{d_{n}(\phi, R+1)}{R}+\frac{d_{n}(\psi, R+1)}{R}\right) \leq 4\left(m+2 n_{0}\right) \frac{m(2 R)}{S(R)}+\log \frac{4}{3} .
$$

If $0<\alpha \leq \beta$, then $\log (\alpha+\beta) \leq \log \beta+\log 2$. Thus

$$
\log \frac{R}{\max \left\{d_{n}(\phi, R+1), d_{n}(\psi, R+1)\right\}} \leq 8\left(n+n_{0}\right) \frac{m(2 R)}{S(R)}+\log 3 .
$$

If $\operatorname{diam}_{m}(F) \geq 1$ then

$$
\max \left\{d_{n}(\phi, R+1), d_{n}(\psi, R+1)\right\} \geq 1 / 2 .
$$

Consequently, if the estimate in the statement of the corollary fails for some $e^{i \phi}$, then it must hold for all $e^{i \psi}$ lying at spherical distance at least 1 from $e^{i \phi}$. Since the set of such $e^{i \psi}$ is an arc of length greater than $\pi$, the corollary follows.

\section{General Estimates FOR $e_{n}$ AND $m_{n}(r)$}

Let $f$ be an entire transcendental function and recall that $n_{0}=n_{0}(f)$ is the maximum of the numbers $\left|f^{-1}(w) \cap \Delta_{2}\right|$, when $|w|=1$. In the following lemma, the estimates on the $n$-th diameter obtained in the previous section, combined with results form [CP2], lead to estimates of the transcendence measures $e_{n}$ and $m_{n}(r)$ in terms of $m(r)$.

Lemma 4.1. Let $R_{0}=R_{0}(f)$ be the largest among the unique solutions of the equations:

$$
R=64, \quad S(R)=\frac{288 \pi^{2}}{\log (4 / 3)}, \quad m(R)=4 \log ^{+} R, \quad m(4 R)=36 .
$$

If $R>R_{0}$ and $n \leq \frac{1}{2} \Lambda S(R)-n_{0}$, then

$$
\begin{aligned}
e_{n} & \leq 2 n m(4 R) \log R, \\
m_{n}(r) & \leq 3 n m(4 R) \log r, 1 \leq r \leq R .
\end{aligned}
$$

Proof. Using Lemma 2.2 with $k=4 / 3$ and $\epsilon=1 / 6$ we can find, for all $R>R_{0}$, a radius $R^{\prime} \in(R, 4 R / 3)$ so that $L\left(R^{\prime}\right) \leq S\left(R^{\prime}\right) / 6$. Since $n+n_{0} \leq \frac{1}{2} \Lambda S(R)$, we have by Corollary 3.4 with $r=R^{\prime}+1$, that for $e^{i \theta}$ in a set of length $l>\pi$ in $\partial \Delta$

$$
\log \frac{R^{\prime}}{d_{n}(\theta, r)} \leq \max \{4 \Lambda m(2 r)+\log 3, \log (2 r)\} .
$$


Since $e r<3 R^{\prime}$, the latter inequality implies

$$
\log \frac{36 e r}{d_{n}(\theta, r)} \leq 5+\max \{4 \Lambda m(2 r)+\log 3, \log (2 r)\} .
$$

Theorem 4.2 in [CP2] asserts that if for some $r \geq 2$ one has $d_{n}(\theta, r) \geq$ $a$ on a set $E \subset \partial \Delta$ of length $l$, then

$$
e_{n} \leq n \max \{m(e r), \log (e r)\} \log r+n \log (e r)\left(\log \frac{36 e r}{a}+\frac{4 \pi}{l}\right) .
$$

Suppose that $4 \Lambda m(2 r)+\log 3 \geq \log (2 r)$. Since $m(e r) \geq \log (e r)$, the above estimate yields

$$
e_{n} \leq n m(e r) \log r+n \log (e r)(11+4 \Lambda m(2 r)) .
$$

Since $e r<4 R, R>64, m(4 R)>36$ and $\Lambda<e^{-300}$ we have

$$
\begin{aligned}
e_{n} & \leq\left(1+4 \Lambda+\frac{11}{m(4 R)}\right) n m(4 R) \log (4 R) \\
& \leq \frac{4}{3}\left(1+4 \Lambda+\frac{11}{36}\right) n m(4 R) \log R<2 n m(4 R) \log R .
\end{aligned}
$$

If $4 \Lambda m(2 r)+\log 3<\log (2 r)$, then using in addition that $4 \log (4 R) \leq$ $m(4 R)$, we get

$$
\begin{aligned}
e_{n} & \leq n m(e r) \log r+n \log (e r)(9+\log (2 r)) \\
& \leq\left(1+\frac{9}{m(4 R)}+\frac{1}{4}\right) n m(4 R) \log (4 R) \leq 2 n m(4 R) \log R .
\end{aligned}
$$

Thus $e_{n} \leq 2 n m(4 R) \log R$. By $[\mathrm{CP} 2, \S 4(5)]$ we have for $1 \leq r \leq R$

$$
m_{n}(r) \leq \frac{e_{n}+n m(R)}{\log R} \log r \leq 3 n m(4 R) \log r .
$$

The above lemma shows that estimates for $e_{n}$ and $m_{n}$ require knowledge of the relationship between $m(4 R)$ and $S(R)$. The following theorem shows the kind of hypotheses on $m(4 R)$ and $S(R)$ needed to get good estimates on $e_{n}$ and $m_{n}$.

We denote by $R_{1}(f)$ the maximum of $R_{0}(f)$ and the solution of the equation

$$
T_{0}(r)=(3 \log 2) / 2+3 \log ^{+}|f(0)| .
$$

We call an interval

$$
I(R, \alpha, \beta, \gamma, C)=\left[\begin{array}{c}
\left.\beta S^{\gamma}(R), \frac{1}{2} \Lambda S(R)-n_{0}\right] \\
13
\end{array}\right]
$$


admissible if $R>R_{1}(f), \alpha, \beta>0,0<\gamma \leq 1, \beta S^{\gamma}(R) \leq \frac{1}{2} \Lambda S(R)-$ $n_{0}-1, S(R) \geq R^{\alpha}$ and $m(4 R) \leq C S(R)$. We let $I(\alpha, \beta, \gamma, C)$ be the union of all admissible intervals $I(R, \alpha, \beta, \gamma, C)$.

Theorem 4.2. If $n \in I(\alpha, \beta, \gamma, C)$, then

$$
e_{n} \leq \frac{2 C}{\alpha \gamma \beta^{1 / \gamma}} n^{1+1 / \gamma} \log \frac{n}{\beta} .
$$

If $R$ is so that $n \in I(R, \alpha, \beta, \gamma, C)$, then $2 \Lambda m(2 R) \geq n$ and

$$
m_{n}(r) \leq \frac{3 C}{\beta^{1 / \gamma}} n^{1+1 / \gamma} \log r, 1 \leq r \leq R .
$$

Proof. By the properties of admissible intervals we have

$$
m(4 R) \leq C S(R) \leq C\left(\frac{n}{\beta}\right)^{1 / \gamma}
$$

and

$$
R \leq S^{1 / \alpha}(R) \leq\left(\frac{n}{\beta}\right)^{1 /(\alpha \gamma)}
$$

By Lemma 4.1

$$
e_{n} \leq \frac{2 C}{\alpha \gamma \beta^{1 / \gamma}} n^{1+1 / \gamma} \log \frac{n}{\beta}
$$

and

$$
m_{n}(r) \leq \frac{3 C}{\beta^{1 / \gamma}} n^{1+1 / \gamma} \log r, 1 \leq r \leq R .
$$

By (1) and (2) we have

$$
T_{0}(r)-\frac{\log 2}{2} \leq m(r) \leq 3 T_{0}(2 r)+\frac{3 \log 2}{2}+3 \log ^{+}|f(0)| .
$$

So if $r \geq R_{1}(f)$ then

$$
\frac{1}{2} T_{0}(r) \leq m(r) \leq 4 T_{0}(2 r) .
$$

By $(3) S(R) \log 2 \leq T_{0}(2 R)$, so

$$
S(R) \leq \frac{2 m(2 R)}{\log 2}
$$

Therefore $n \leq \Lambda S(R) / 2 \leq 2 \Lambda m(2 R)$, and the proof is complete.

The following corollary establishes a case when polynomial estimates for $e_{n}$ hold for all $n$.

Corollary 4.3. Suppose that there is a sequence of admissible intervals $I\left(R_{j}, \alpha, \beta, \gamma, C\right)$ such that $R_{j} \rightarrow \infty$ and $\beta S^{\gamma}\left(R_{j+1}\right) \leq \Lambda S\left(R_{j}\right) / 2-n_{0}$, $j \geq 1$. Then the conclusions of Theorem 4.2 hold for all $n \geq \beta S^{\gamma}\left(R_{1}\right)$. 
A system of admissible intervals satisfying the hypotheses of this corollary will be called a covering system.

\section{THE LOWER ORDER OF TRANSCENDENCE}

In order to apply Theorem 4.2 effectively we need information on the set $I(\alpha, \beta, \gamma, C)$. The main goal of this section is to establish that for every entire function of finite positive order we can find $\alpha, \beta$ and $C$ such that the set $I(\alpha, \beta, 1, C)$ is unbounded. Then Theorem 4.2 will imply that the lower order of transcendence $\underline{\tau}(f)$ of any entire function $f$ of finite positive order is 2 . Our first step is to study the ratio of $T_{0}(r)$ and $S(r)$.

Lemma 5.1. If $A T_{0}\left(r_{1} / k\right) \leq T_{0}\left(r_{1}\right)$, where $k>1$ and $A>1$, then there is $r \in\left(r_{1} / k, r_{1}\right)$ such that $c S(r) \geq T_{0}\left(r_{1}\right)$, where

$$
c=\frac{A \log k}{\log A} \text {. }
$$

Proof. Let us take $r_{2}$ such that $T_{0}\left(r_{1}\right)=A T_{0}\left(r_{2}\right)$. Then $r_{1} / k \leq r_{2}<r_{1}$. If

$$
T_{0}(t)>\frac{\log k}{\log A} S(t)
$$

on $\left(r_{2}, r_{1}\right)$, then

$$
\log A=\int_{r_{2}}^{r_{1}} \frac{T_{0}^{\prime}(t)}{T_{0}(t)} d t=\int_{r_{2}}^{r_{1}} \frac{S(t)}{t T_{0}(t)} d t<\frac{\log A}{\log k} \int_{r_{2}}^{r_{1}} \frac{d t}{t} \leq \log A .
$$

Hence there is $r \in\left(r_{2}, r_{1}\right)$ such that

$$
\frac{\log k}{\log A} S(r) \geq T_{0}(r) \geq T_{0}\left(r_{2}\right)=\frac{T_{0}\left(r_{1}\right)}{A} .
$$

Next we need the ratio $m(4 r) / S(r)$ to be bounded above for some numbers $r$. The following lemma provides sufficient conditions for such values of $r$.

Lemma 5.2. Suppose that for some $k>1$ and $r_{1}>k R_{1}(f)$ there are constants $A_{1}>8$ and $A_{2}>1$ such that $A_{1} m\left(r_{1} / k\right) \leq m\left(r_{1}\right)$ and $m\left(8 r_{1}\right) \leq A_{2} m\left(r_{1}\right)$. Then there is $r \in\left(r_{1} / k, 2 r_{1}\right)$ such that $C S(r) \geq$ $m\left(8 r_{1}\right)$, where

$$
C=\frac{A_{1} A_{2} \log (2 k)}{2 \log \left(A_{1} / 8\right)}
$$


Proof. By (7) it follows that

$$
\frac{1}{2} T_{0}\left(r_{1} / k\right) \leq m\left(r_{1} / k\right) \leq \frac{m\left(r_{1}\right)}{A_{1}} \leq \frac{4 T_{0}\left(2 r_{1}\right)}{A_{1}} .
$$

By Lemma 5.1 there is $r \in\left(r_{1} / k, 2 r_{1}\right)$, such that $c_{1} S(r) \geq T_{0}\left(2 r_{1}\right)$, where

$$
c_{1}=\frac{A_{1} \log (2 k)}{8 \log \left(A_{1} / 8\right)} .
$$

Hence

$$
c_{1} S(r) \geq T_{0}\left(2 r_{1}\right) \geq \frac{m\left(r_{1}\right)}{4} \geq \frac{m\left(8 r_{1}\right)}{4 A_{2}} .
$$

As we will now see, the ratio $m(4 r) / S(r)$ is bounded near points where $m(r)$ is close to its proximate order.

Lemma 5.3. Suppose that $m(r) \leq r^{\phi(r)}$, where $\lim _{r \rightarrow \infty} \phi(r)=\rho$ and the function $r^{\phi(r)-\rho}$ is slowly increasing. Let $0<a \leq 1$ and $k^{\rho}>$ 16/a. If $r_{1}$ is sufficiently large and $m\left(r_{1}\right) \geq a r_{1}^{\phi\left(r_{1}\right)}$, then there is $r \in\left(r_{1} / k, 2 r_{1}\right)$ such that $C S(r) \geq m\left(8 r_{1}\right)$, where

$$
C=\frac{(8 k)^{\rho} \log (2 k)}{2 \log \left(a k^{\rho} / 16\right)} .
$$

Proof. "Sufficiently large" in the statement of the lemma means $r_{1}>$ $k r_{0}$, where $r_{0}>R_{1}(f)$ is a number such that

$$
\frac{1}{2} b^{\rho} r^{\phi(r)}<(b r)^{\phi(b r)}<2 b^{\rho} r^{\phi(r)}
$$

holds for $k^{-1} \leq b \leq 8$ and $r \geq r_{0}$ (see (5)). Then

$$
m\left(r_{1} / k\right) \leq\left(\frac{r_{1}}{k}\right)^{\phi\left(r_{1} / k\right)} \leq 2 k^{-\rho} r_{1}^{\phi\left(r_{1}\right)} \leq \frac{2}{a k^{\rho}} m\left(r_{1}\right),
$$

and

$$
m\left(8 r_{1}\right) \leq\left(8 r_{1}\right)^{\phi\left(8 r_{1}\right)} \leq 2^{3 \rho+1} r_{1}^{\phi\left(r_{1}\right)} \leq \frac{2^{3 \rho+1}}{a} m\left(r_{1}\right) .
$$

The conclusion follows by Lemma 5.2, if we let $A_{1}=a k^{\rho} / 2$ and $A_{2}=$ $2^{3 \rho+1} / a$.

In the following theorem we prove that $\underline{\tau}(f)=2$. Note that we also give effective estimates on the "type" of growth of $e_{n}$ and $m_{n}(r)$. 
Theorem 5.4. Let $f$ be an entire function of finite order $\rho>0$. There exist sequences of integers $n_{j} \nearrow \infty$ and $\epsilon_{j} \rightarrow 0, \epsilon_{j}>0$, such that

$$
\begin{aligned}
& \frac{n_{j}^{2} \log n_{j}}{2 \rho+1} \leq e_{n_{j}} \leq \frac{8^{\rho+3}(\rho+5)}{\Lambda \rho^{2}} n_{j}^{2} \log n_{j}, \\
& m_{n_{j}}(r) \leq \frac{8^{\rho+3}(\rho+5)}{\Lambda \rho} n_{j}^{2} \log r, 1 \leq r \leq \frac{1}{2} n_{j}^{1 / \rho-\epsilon_{j}} .
\end{aligned}
$$

Proof. Let $\rho(r)$ be a proximate order for $m(r)$. By its definition there exists a sequence $R_{j}^{\prime} \rightarrow \infty$ such that $m\left(R_{j}^{\prime}\right)=\left(R_{j}^{\prime}\right)^{\rho\left(R_{j}^{\prime}\right)}$. Take $a=1$ and $k=2^{5 / \rho}$. By Lemma 5.3 there exist, for all $j$ sufficiently large, numbers $R_{j} \in\left(R_{j}^{\prime} / k, 2 R_{j}^{\prime}\right)$ such that

$$
C S\left(R_{j}\right) \geq m\left(8 R_{j}^{\prime}\right) \geq m\left(4 R_{j}\right),
$$

where

$$
C=\frac{2^{3 \rho+4}(\rho+5)}{\rho} .
$$

Since for $j$ large

$$
m\left(8 R_{j}^{\prime}\right)>\left(R_{j}^{\prime}\right)^{\rho\left(R_{j}^{\prime}\right)}>\left(R_{j} / 2\right)^{3 \rho / 4},
$$

we see that $S\left(R_{j}\right) \geq R_{j}^{\rho / 2}$. Also $S\left(R_{j}\right) \geq 6\left(n_{0}+1\right) / \Lambda$ when $j$ is sufficiently large.

Hence the intervals $I_{j}=I\left(R_{j}, \rho / 2, \Lambda / 3,1, C\right)$ are admissible and there exists a sequence of integers $n_{j} \in I_{j}$. By Theorem 4.2

$$
e_{n_{j}} \leq \frac{3 \cdot 2^{3 \rho+6}(\rho+5)}{\Lambda \rho^{2}} n_{j}^{2} \log \frac{3 n_{j}}{\Lambda} \leq \frac{2^{3 \rho+9}(\rho+5)}{\Lambda \rho^{2}} n_{j}^{2} \log n_{j},
$$

for all $j$ sufficiently large. Moreover

$$
m_{n_{j}}(r) \leq \frac{2^{3 \rho+8}(\rho+5)}{\Lambda \rho} n_{j}^{2} \log r, 1 \leq r \leq R_{j},
$$

and

$$
n_{j} \leq 2 \Lambda m\left(2 R_{j}\right) \leq 2 \Lambda\left(2 R_{j}\right)^{\rho\left(2 R_{j}\right)} .
$$

By (5) there is a sequence of positive $\epsilon_{j} \rightarrow 0$ such that

$$
n_{j} \leq 2 \Lambda\left(2 R_{j}\right)^{\rho\left(2 R_{j}\right)} \leq 4 \Lambda 2^{\rho} R_{j}^{\rho\left(R_{j}\right)} \leq 2^{\rho} R_{j}^{1 /\left(1 / \rho-\epsilon_{j}\right)} .
$$

Hence $R_{j} \geq n_{j}^{1 / \rho-\epsilon_{j}} / 2$.

For the lower estimate on $e_{n_{j}}$, we take $r$ with $n_{j}=m(r)<r^{\rho+1 / 4}$, so $\log r>\log n_{j} /(\rho+1 / 4)$. By [CP2, $\$ 4$ (3)] and [CP2, Corollary 2.6]

$$
e_{n_{j}} \geq \frac{n_{j}^{2}}{2} \log r-n_{j} m(r) \geq \frac{n_{j}^{2} \log n_{j}}{2 \rho+1 / 2}-n_{j}^{2} \geq \frac{n_{j}^{2} \log n_{j}}{2 \rho+1} .
$$




\section{Doubling inequalities}

In this section we prove doubling inequalities, which provide upper bounds for the ratio $M(2 r, F) / M(r, F)$, where $F(z)=P(z, f(z))$ and $P$ is a polynomial. For $f(z)=e^{z}$ such inequalities were obtained by Tijdeman in [Ti1].

We will need a simple lemma, whose proof is contained in the proof of Theorem 2.2 of [CP2].

Lemma 6.1. If $r<s$ and an entire function $f$ has $m$ zeros in $\Delta_{r}$, then

$$
\frac{M(s, f)}{M(r, f)} \geq\left(\frac{r^{2}+s^{2}}{2 r s}\right)^{m}
$$

First, we reduce the problem of doubling inequalities to the problem of obtaining estimates for the transcendence measures $m_{n}$ of dilations $f_{r}(z)=f(r z)$ of $f$. Let $r \geq 1$, let $P(z, w)$ be a polynomial of degree at most $n$ and let $F(z)=P(z, f(z))$. If $P_{r}(z, w)=P(r z, w)$, then $\left\|P_{r}\left(z, f_{r}(z)\right)\right\|_{\Delta}=M(r, F)$, while $\left\|P_{r}\left(z, f_{r}(z)\right)\right\|_{\Delta_{2}}=M(2 r, F)$. Hence

$$
\frac{M(2 r, F)}{M(r, F)} \leq e^{m_{n}\left(2, f_{r}\right)}
$$

and we have to estimate $m_{n}\left(2, f_{r}\right)$.

Theorem 6.2. Let $f$ be an entire transcendental function of finite positive order $\rho$. There exists a sequence of integers $\left\{n_{j}\right\}$ increasing to infinity with the following property: For every $r \geq 1$ there is an integer $j_{r}$ such that

$$
\frac{M(2 r, F)}{M(r, F)} \leq 2^{a n_{j}^{2}}, \quad a=\frac{8^{\rho+3}(\rho+5)}{\Lambda \rho}, \quad j \geq j_{r},
$$

where $F(z)=P(z, f(z))$ and $P(z, w)$ is a polynomial of degree at most $n_{j}$.

Proof. Let us denote by $n_{r}$ the maximum of the numbers $\left|f_{r}^{-1}(w) \cap \Delta_{2}\right|$, when $|w|=1$. Let $w_{r}$ be a point where this maximum is achieved and let $g_{r}(z)=f(r z)-w_{r}$. By Lemma 6.1

$$
\left(\frac{5}{4}\right)^{n_{r}} \leq \frac{M\left(4, g_{r}\right)}{M\left(2, g_{r}\right)} .
$$

Since $f$ is not constant, there exists $\epsilon>0$ such that $M\left(2, g_{r}\right) \geq \epsilon$ for every $r \geq 1$. Since $M\left(4, g_{r}\right) \leq M(4 r)+1$ it follows that

$$
n_{r} \leq C_{1} m(4 r)-1
$$


where $C_{1}$ is a constant depending only on $f$.

Let $I=I(R, \alpha, \beta, \gamma, C)$ be an admissible interval for $f$. From the definition of the number $R_{1}(f)$ in Section 4 it follows that $R_{1}\left(f_{r}\right) \leq$ $R_{1}(f)$. Note that $m\left(t, f_{r}\right)=m(r t)$ and $S_{f_{r}}(t)=S(r t)$. Hence, if $R^{\prime}=R / r$, then $S_{f_{r}}\left(R^{\prime}\right) \geq R^{\prime \alpha}$ and $m\left(4 R^{\prime}, f_{r}\right) \leq C S_{f_{r}}\left(R^{\prime}\right)$. Therefore, the interval $I^{\prime}=\left(R^{\prime}, \alpha, \beta, \gamma, C\right)$ is admissible for $f_{r}$ if $R^{\prime} \geq R_{1}(f)$ and

$$
\frac{\Lambda}{2} S(R)-n_{r}-1 \geq \beta S^{\gamma}(R) .
$$

Since $f$ has finite positive order $\rho$, by the proof of Theorem 5.4 there is a sequence $R_{j}$ increasing to infinity such that the intervals

$$
I_{j}=I\left(R_{j}, \rho / 2, \Lambda / 3,1, C\right)=\left[\frac{\Lambda S\left(R_{j}\right)}{3}, \frac{\Lambda S\left(R_{j}\right)}{2}-n_{0}\right]
$$

are admissible, where $C$ is defined in (9). For every $r \geq 1$ let $j_{r}$ be the smallest integer such that $R_{j_{r}}>r R_{1}(f)$ and

$$
\frac{\Lambda}{10} S\left(R_{j_{r}}\right) \geq C_{1} m(4 r) \text {. }
$$

Then

$$
\frac{\Lambda}{2} S\left(R_{j_{r}}\right)-n_{r}-1 \geq \frac{2 \Lambda}{5} S\left(R_{j_{r}}\right) \geq \frac{\Lambda}{3} S\left(R_{j_{r}}\right)
$$

and the intervals

$$
I_{j}^{\prime}=I\left(R_{j} / r, \rho / 2, \Lambda / 3,1, C\right)=\left[\frac{\Lambda S\left(R_{j}\right)}{3}, \frac{\Lambda S\left(R_{j}\right)}{2}-n_{r}\right]
$$

are admissible for $f_{r}$ when $j \geq j_{r}$.

Let $j_{0}$ be the smallest integer so that $S\left(R_{j_{0}}\right) \geq \max \left\{15 / \Lambda, 10 n_{0} / \Lambda\right\}$. Then for $j \geq j_{0}$ the intervals $I_{j}^{\prime \prime}=\left[\Lambda S\left(R_{j}\right) / 3,2 \Lambda S\left(R_{j}\right) / 5\right]$ contain an integer $n_{j}$ and $I_{j}^{\prime \prime} \subset I_{j}$. Moreover, if $j \geq j_{r}$ then $I_{j}^{\prime \prime} \subset I_{j}^{\prime}$, so by Theorem 4.2

$$
m_{n_{j}}\left(2, f_{r}\right) \leq \frac{9 C}{\Lambda} n_{j}^{2} \log 2 \leq a n_{j}^{2} \log 2 .
$$

Consequently $M(2 r, F) / M(r, F) \leq 2^{a n_{j}^{2}}$, for all $j \geq j_{r}$.

Remark. With the notations of the above proof, since $n_{j} \in I_{j}^{\prime \prime} \subset I_{j}$ it follows that the conclusions of Theorem 5.4 hold for the sequence $\left\{n_{j}\right\}$ constructed in Theorem 6.2. A sequence of integers $\left\{n_{j}\right\}$ increasing to infinity for which the conclusions of both Theorems 5.4 and 6.2 are valid, will be called a fundamental sequence for $f$.

In the following theorem we prove doubling inequalities for functions which possess a covering system of admissible intervals. 
Theorem 6.3. Let $f$ be an entire transcendental function which has a covering system of admissible intervals $I_{j}=I\left(R_{j}, \alpha, \beta, \gamma, C\right)$. For every $r \geq 1$ there exists an integer $j_{r}$ such that

$$
\frac{M(2 r, F)}{M(r, F)} \leq\left\{\begin{array}{l}
\exp \left(3 n m\left(4 R_{j_{r}}\right) \log 2\right), \text { if } n<\beta S^{\gamma}\left(R_{j_{r}}\right) / 2, \\
\exp \left(3 C\left(2 \beta^{-1}\right)^{1 / \gamma} n^{1+1 / \gamma} \log 2\right), \text { if } n \geq \beta S^{\gamma}\left(R_{j_{r}}\right) / 2,
\end{array}\right.
$$

where $F(z)=P(z, f(z))$ and $P(z, w)$ is a polynomial of degree at most $n$.

Proof. Let $j_{r}$ be the smallest integer such that

$$
R_{j_{r}}>r R_{1}(f), \Lambda m\left(4 R_{j_{r}}\right) \geq 4 C C_{1} m(4 r),
$$

where $C_{1}$ is the constant from (10). By (10) and the properties of admissible intervals we have for $j \geq j_{r}$

SO

$$
\frac{\Lambda}{4} S\left(R_{j}\right) \geq \frac{\Lambda}{4 C} m\left(4 R_{j_{r}}\right) \geq C_{1} m(4 r) \geq n_{r}+1
$$

$$
\frac{\Lambda}{2} S\left(R_{j}\right)-n_{r}-1 \geq \frac{\Lambda}{4} S\left(R_{j}\right) \geq \frac{\beta}{2} S^{\gamma}\left(R_{j}\right) .
$$

Moreover, since $I_{j}$ form a covering system we have

$$
\frac{\beta}{2} S^{\gamma}\left(R_{j+1}\right) \leq \frac{\Lambda}{4} S\left(R_{j}\right)-\frac{n_{0}}{2} \leq \frac{\Lambda}{2} S\left(R_{j}\right)-n_{r}
$$

Thus the intervals $I_{j}^{\prime}=I\left(R_{j} / r, \alpha, \beta / 2, \gamma, C\right), j \geq j_{r}$, form a covering system of admissible intervals for $f_{r}$. By Corollary 4.3

$$
m_{n}\left(2, f_{r}\right) \leq 3 C\left(2 \beta^{-1}\right)^{1 / \gamma} n^{1+1 / \gamma} \log 2,
$$

when $n \geq \beta S^{\gamma}\left(R_{j_{r}}\right) / 2$.

If

$$
n<\beta S^{\gamma}\left(R_{j_{r}}\right) / 2 \leq \frac{\Lambda}{2} S\left(R_{j_{r}}\right)-n_{r},
$$

then by Lemma $4.1 m_{n}\left(2, f_{r}\right) \leq 3 n m\left(4 R_{j_{r}}\right) \log 2$.

Let us denote by $Z_{n}(r, f)=Z_{n}(r)$ the maximal number of zeros of $P(z, f(z))$ in $\Delta_{r}$, when $P(z, w)$ is a polynomial of degree at most $n$. In Corollary 2.6 of [CP2] we proved that $Z_{n}(r) \leq 2 m_{n}(3 r)$. Now we can improve this estimate.

The first result gives an estimate on $Z_{n}(r)$ for all transcendental functions of finite positive order. Note that the constant $a$ depends only on the order $\rho$ of $f$.

Corollary 6.4. If $\left\{n_{j}\right\}$ is a fundamental sequence for $f$ then $Z_{n_{j}}(r) \leq$ $4 a n_{j}^{2}$, for $r \geq 1$ and $j \geq j_{r}$. 
Proof. Let $P(z, w)$ be a polynomial of degree $n_{j}$ such that the number of zeros of $F(z)=P(z, f(z))$ in $\Delta_{r}$ equals $Z_{n_{j}}(r)$. Then by Theorem 6.2 and Lemma 6.1

$$
\left(\frac{5}{4}\right)^{Z_{n_{j}}(r)} \leq \frac{M(2 r, F)}{M(r, F)} \leq 2^{a n_{j}^{2}}
$$

when $j \geq j_{r}$. Hence

$$
Z_{n_{j}}(r) \leq \frac{a n_{j}^{2} \log 2}{\log (5 / 4)} \leq 4 a n_{j}^{2} .
$$

The second corollary provides estimates on $Z_{n}(r)$ for all $n$ and has a similar proof.

Corollary 6.5. In the assumptions of Theorem 6.3 we have

$$
Z_{n}(r) \leq\left\{\begin{array}{l}
10 n m\left(4 R_{j_{r}}\right), \text { if } n<\beta S^{\gamma}\left(R_{j_{r}}\right) / 2, \\
10 C\left(2 \beta^{-1}\right)^{1 / \gamma} n^{1+1 / \gamma}, \text { if } n \geq \beta S^{\gamma}\left(R_{j_{r}}\right) / 2 .
\end{array}\right.
$$

Doubling inequalities lead to tangential Markov inequalities, which provide upper estimates for the derivative of the function $F(z)=$ $P(z, f(z))$, where $P(z, w)$ is a polynomial of degree $n$. As before, we give two versions of such inequalities: one for general entire functions and another for functions with a covering system of admissible intervals.

Theorem 6.6. Let $\left\{n_{j}\right\}$ be a fundamental sequence for $f$. For every $r \geq 1$ there is an integer $j_{r}$ such that

$$
M\left(r, F^{\prime}\right) \leq \frac{e a M(r, F) n_{j}^{2}}{r},
$$

where $F(z)=P(z, f(z)), P(z, w)$ is a polynomial of degree $n_{j}$, and $j \geq j_{r}$.

Proof. For $r \geq 1$ let $j_{r}$ be the integer from Theorem 6.2. Let $F(z)=$ $P(z, f(z))$, where $P(z, w)$ is a polynomial of degree $n_{j}$ and $j \geq j_{r}$. Since $m(r, F)$ is a convex increasing function of $\log r$

$$
|F(z)| \leq M(r, F) \exp \left((m(t, F)-m(r, F)) \frac{\log (|z| / r)}{\log (t / r)}\right), r \leq|z| \leq t .
$$

Let $b>1$ be such that

$$
\frac{m(t, F)-m(r, F)}{\log (t / r)} \leq b .
$$


The function $h(x)=e^{b \log (1+x)} / x$ attains its minimum value when $x=$ $x_{b}=1 /(b-1)$, and $h\left(x_{b}\right)<e b$. Therefore, if $r\left(1+x_{b}\right) \leq t$ and $|z|=r$, then by the Cauchy estimates

$$
\left|F^{\prime}(z)\right| \leq \frac{M(r, F)}{r x_{b}} e^{b \log \left(1+x_{b}\right)} \leq \frac{e b M(r, F)}{r} .
$$

Taking $t=2 r$, we have by Theorem $6.2(m(2 r, F)-m(r, F)) / \log 2 \leq$ $b=a n_{j}^{2}$ and $1+x_{b} \leq 2$. Thus

$$
\left|F^{\prime}(z)\right| \leq \frac{\operatorname{eaM}(r, F) n_{j}^{2}}{r}
$$

The following theorem provides estimates on $M\left(r, F^{\prime}\right)$ for all $n$ and has a similar proof.

Theorem 6.7. In the assumptions of Theorem 6.3 we have

$$
M\left(r, F^{\prime}\right) \leq \frac{3 e C 2^{1 / \gamma} n^{1+1 / \gamma} M(r, F)}{\beta^{1 / \gamma} r},
$$

where $F(z)=P(z, f(z)), P(z, w)$ is a polynomial of degree $n$, and $n \geq \beta S^{\gamma}\left(R_{j_{r}}\right) / 2$.

\section{Special Classes of FUnCtions}

In this section we find sufficient conditions for a function $f$ to have estimates of the form $e_{n}=O\left(n^{\tau} \log n\right)$ for some $\tau \geq 2$. These conditions are imposed on the growth of $f$ and are easy to verify. We start with the class of entire functions $f$ whose growth satisfies the following inequalities: There exist constants $A_{2}>A_{1}>1$ and $k>1$ such that

$$
A_{1} m(r) \leq m(k r) \leq A_{2} m(r)
$$

for all $r$ sufficiently large. These are functions of finite positive order and this class includes, for example, all functions

$$
f(z)=\sum_{j=1}^{m} p_{j}(z) e^{q_{j}(z)},
$$

where $p_{j}$ and $q_{j}$ are polynomials. Moreover we show in the next section that the Riemann $\zeta$-function and the function $\xi$ are also in this class.

Theorem 7.1. Let $f$ be an entire function of order $\rho$ which satisfies (12) for all $r$ sufficiently large. Then, for all $n$ sufficiently large,

$$
e_{n} \leq K_{1} n^{2} \log n, m_{n}(r) \leq K_{2} n^{2} \log r, 1 \leq r \leq n^{1 / \rho-\epsilon_{n}} / 2,
$$

where the constants $K_{1}, K_{2}$ depend only on $A_{1}, A_{2}, k$, and $\epsilon_{n}>0, \epsilon_{n} \rightarrow$ 0 . 
Proof. Inequalities (12) imply that

$$
A_{1}^{j} m(r) \leq m\left(k^{j} r\right) \leq A_{2}^{j} m(r), d_{1} r^{\rho_{1}} \leq m(r) \leq d_{2} r^{\rho_{2}},
$$

where

$$
\rho_{1}=\frac{\log A_{1}}{\log k}, \rho_{2}=\frac{\log A_{2}}{\log k}, d_{1}=\frac{m(1)}{A_{1}}, d_{2}=A_{2} m(1) .
$$

Thus $f$ is a function of finite positive order $\rho \in\left[\rho_{1}, \rho_{2}\right]$, and we may assume that (12) holds with constants $k \geq 8$ and $A_{1}>8$. Then $A_{1} m(r / k) \leq m(r)$ and $m(8 r) \leq A_{2} m(r)$.

For every $r$ sufficiently large there is, by Lemma 5.2, $r^{\prime} \in(r / k, 2 r)$ such that $C S\left(r^{\prime}\right) \geq m\left(4 r^{\prime}\right)$, where

$$
C=\frac{A_{1} A_{2} \log (2 k)}{2 \log \left(A_{1} / 8\right)} .
$$

In particular, for all $j$ sufficiently large, there is $R_{j} \in\left((2 k)^{j},(2 k)^{j+1}\right)$ such that $C S\left(R_{j}\right) \geq m\left(4 R_{j}\right)$. Since the order of $f$ is $\rho$ we may assume that $S\left(R_{j}\right) \geq R_{j}^{\rho / 2}$.

Using (8) and (12) we get

$$
S\left(R_{j+1}\right) \leq 3 m\left(2 R_{j+1}\right) \leq 3 m\left(8 k^{2}(2 k)^{j}\right) \leq 3 A_{2}^{3} m\left(R_{j}\right) \leq 3 C A_{2}^{3} S\left(R_{j}\right) .
$$

Hence $S\left(R_{j+1}\right) / S\left(R_{j}\right) \leq M=3 C A_{2}^{3}$.

Let $j$ be so large that $S\left(R_{j}\right) \geq 6\left(n_{0}+1\right) / \Lambda$ and let $\beta=\Lambda /(3 M)$. Then

$$
\beta S\left(R_{j}\right) \leq \beta S\left(R_{j+1}\right) \leq \frac{\Lambda}{3} S\left(R_{j}\right) \leq \frac{\Lambda}{2} S\left(R_{j}\right)-n_{0}-1,
$$

so the intervals $I_{j}=I\left(R_{j}, \rho / 2, \beta, 1, C\right)$ form a covering system of admissible intervals, starting with some $j$ sufficiently large. The theorem now follows from Corollary 4.3. If $n \in I_{j_{n}}$ then the fact that $R_{j_{n}} \geq n^{1 / \rho-\epsilon_{n}} / 2$ can be proved exactly like the similar statement in Theorem 5.4.

The functions $f$ satisfying (12) have covering systems of admissible intervals. Hence they also satisfy the hypotheses of Theorem 6.3. Moreover, in this case we can get better estimates on the integers $j_{r}$ from Theorem 6.3.

Corollary 7.2. Let $f$ be an entire function of order $\rho$ which satisfies (12) for all $r$ sufficiently large. Then there is a constant $a>1$ such that $Z_{n}(r) \leq a\left(n m(\right.$ ar $\left.)+n^{2}\right)$, for all $n \geq 1$ and $r \geq 1$.

Proof. Fix $r \geq 1$, let $I_{j}=I\left(R_{j}, \rho / 2, \beta, 1, C\right)$ be the covering system of admissible intervals from the proof of Theorem 7.1, and recall that $R_{j} \in$ $\left((2 k)^{j},(2 k)^{j+1}\right)$. By Corollary 6.5 we have $Z_{n}(r) \leq 10 n m\left(4 R_{j_{r}}\right)+A n^{2}$ 
for all $n \geq 1$, where $A$ is a constant and $j_{r}$ is defined in (11) as the smallest integer such that $R_{j_{r}}>r R_{1}(f)$ and $\Lambda m\left(4 R_{j_{r}}\right) \geq 4 C C_{1} m(4 r)$.

We fix $j_{0}, j_{1}$ such that

$$
(2 k)^{j_{0}} \geq R_{1}(f), A_{1}^{j_{0}} \geq 4 C C_{1} / \Lambda, r \in\left[(2 k)^{j_{1}},(2 k)^{j_{1}+1}\right) .
$$

Then $R_{j_{0}+j_{1}+1}>r R_{1}(f)$ and

$$
m\left(4 R_{j_{0}+j_{1}+1}\right) \geq A_{1}^{j_{0}} m\left(4(2 k)^{j_{1}+1}\right)>\frac{4 C C_{1}}{\Lambda} m(4 r) .
$$

Consequently, $j_{r} \leq j_{0}+j_{1}+1, R_{j_{r}} \leq(2 k)^{j_{0}+j_{1}+2} \leq(2 k)^{j_{0}+2} r$, and the corollary follows.

Given an entire function $f$, it is frequently known that $f$ verifies a growth condition $m(r) \leq r^{\phi(r)}$, where $\lim _{r \rightarrow \infty} \phi(r)=\rho$ and the function $r^{\phi(r)-\rho}$ is slowly increasing. In the remainder of this section, we denote by $r_{n}$ the unique solution of the equation $r^{\phi(r)}=n$. Our next theorem shows that in this case there are estimates $e_{n}=O\left(n^{\tau} \log n\right)$, provided that

$$
m\left(r_{n_{j}}\right) \geq a r_{n_{j}}^{\phi\left(r_{n_{j}}\right)}=a n_{j}
$$

holds for a "slow growing" subsequence $n_{j}$.

Theorem 7.3. In the above setting, assume there is an increasing sequence of integers $n_{j}$ such that $n_{j+1}^{\gamma} \leq b n_{j}$ and $m\left(r_{n_{j}}\right) \geq a n_{j}$, where $0<\gamma \leq 1, b>0,0<a \leq 1$. Then there exists a sequence of positive $\epsilon_{n} \rightarrow 0$, such that the estimates

$$
\begin{aligned}
e_{n} & \leq \frac{4 C(3 M)^{1 / \gamma}}{\rho \gamma \Lambda^{1 / \gamma}} n^{1+1 / \gamma} \log \frac{3 M n}{\Lambda} \\
m_{n}(r) & \leq \frac{3 C(3 M)^{1 / \gamma}}{\Lambda^{1 / \gamma}} n^{1+1 / \gamma} \log r, 1 \leq r \leq \frac{1}{2} n^{1 / \rho-\epsilon_{n}},
\end{aligned}
$$

hold for all $n$ sufficiently large, where

$$
C=\frac{2^{3 \rho+4}}{a}\left(1+\frac{1}{\rho} \log _{2}(32 / a)\right), \quad M=\frac{2^{(2 \rho+3) \gamma} C b}{a} .
$$

Proof. We let $s_{j}=r_{n_{j}}$. By Lemma 5.3 with $k=(32 / a)^{1 / \rho}$ and $j$ sufficiently large, there is $R_{j} \in\left(s_{j} / k, 2 s_{j}\right)$ such that

$$
C S\left(R_{j}\right) \geq m\left(8 s_{j}\right) \geq m\left(4 R_{j}\right),
$$

where

$$
C=\frac{2^{3 \rho+4}}{a}\left(1+\frac{1}{\rho} \log _{2}(32 / a)\right) .
$$

We may assume that

$$
\left(4 s_{j}\right)^{\phi\left(4 s_{j}\right)} \leq \underset{24}{2 \cdot 4^{\rho} s_{j}^{\phi\left(s_{j}\right)}}=2^{2 \rho+1} n_{j} .
$$


Using this and (8) we get

$$
S\left(R_{j}\right) \leq \frac{2 m\left(2 R_{j}\right)}{\log 2} \leq 4 m\left(4 s_{j}\right) \leq 2^{2 \rho+3} n_{j}
$$

Since $C S\left(R_{j}\right) \geq m\left(8 s_{j}\right) \geq a n_{j}$ it follows that

$$
\frac{S^{\gamma}\left(R_{j+1}\right)}{S\left(R_{j}\right)} \leq \frac{2^{(2 \rho+3) \gamma} C n_{j+1}^{\gamma}}{a n_{j}} \leq \frac{2^{(2 \rho+3) \gamma} C b}{a}=M .
$$

Moreover,

$$
S\left(R_{j}\right) \geq \frac{a}{C} s_{j}^{\phi\left(s_{j}\right)} \geq \frac{a}{C}\left(R_{j} / 2\right)^{3 \rho / 4} \geq R_{j}^{\rho / 2},
$$

when $j$ is sufficiently large, and if $\beta=\Lambda /(3 M)$ then

$$
\max \left\{\beta S^{\gamma}\left(R_{j}\right), \beta S^{\gamma}\left(R_{j+1}\right)\right\} \leq \frac{\Lambda}{3} S\left(R_{j}\right) \leq \frac{\Lambda}{2} S\left(R_{j}\right)-n_{0}-1 .
$$

So the intervals $I_{j}=I\left(R_{j}, \rho / 2, \beta, \gamma, C\right)$ form a covering system of admissible intervals, starting with some $j$ sufficiently large. The theorem now follows from Corollary 4.3. If $n \in I_{j_{n}}$ then the fact that $R_{j_{n}} \geq n^{1 / \rho-\epsilon_{n}} / 2$ can be proved exactly like the similar statement in Theorem 5.4.

This theorem has a corollary which allows us to estimate $e_{n}$ and $m_{n}$ using the behavior of the Taylor coefficients of $f$.

Corollary 7.4. Suppose that for an entire function

$$
f(z)=\sum_{n=0}^{\infty} c_{n} z^{n}
$$

we have $m(r) \leq r^{\phi(r)}$, where $\lim _{r \rightarrow \infty} \phi(r)=\rho$ and the function $r^{\phi(r)-\rho}$ is slowly increasing. Let $r_{n}$ be defined by $r_{n}^{\phi\left(r_{n}\right)}=n$. If there is an increasing sequence of integers $n_{j}$ such that

$$
n_{j+1}^{\gamma} \leq b n_{j}, \quad \log \left|c_{n_{j}}\right| \geq a n_{j}-n_{j} \log r_{n_{j}},
$$

where $0<\gamma \leq 1, b>0,0<a \leq 1$, then the estimates on $e_{n}$ and $m_{n}(r)$ from Theorem 7.3 hold for all $n$ sufficiently large.

Proof. This follows from Theorem 7.3, since by the Cauchy inequalities we have $m\left(r_{n_{j}}\right) \geq \log \left|c_{n_{j}}\right|+n_{j} \log r_{n_{j}} \geq a n_{j}$.

As an example we take the entire function

$$
f(z)=\sum_{j=1}^{\infty}\left(z / n_{j}\right)^{n_{j}}
$$

where $n_{1} \geq 2, n_{j+1}=n_{j}^{\tau-1}$ and $\tau>2$. This function was studied in Section 6 of [CP2], where it was shown that there are constants $C_{1}$ and 
$C_{2}$ such that $e_{n} \leq C_{1} n^{\tau} \log n$ and $m_{n}(r) \leq C_{2} n^{\tau} \log r$ for $1 \leq r \leq n$. This was a result of quite elaborate estimates. Since $f$ is a function of order 1 and type $1 / e$, we have $m(r) \leq 2 r / e$ for $r$ large. Taking $r_{n_{j}}=e n_{j} / 2$ and $a=\log (e / 2)$ we get

$$
\log c_{n_{j}}=-n_{j} \log n_{j}=a n_{j}-n_{j} \log r_{n_{j}} .
$$

So Corollary 7.4 applies with $\gamma=1 /(\tau-1)$ and $b=1$.

\section{The FunCtions $\zeta$ And $\xi$}

The Riemann $\zeta$-function is holomorphic in $\mathbb{C}$ except at $z=1$, where it has a simple pole (see e.g. [T, Theorem 2.1]). The function $\xi$ is defined by

$$
\xi(z)=\frac{z(z-1)}{2} \pi^{-z / 2} \Gamma\left(\frac{z}{2}\right) \zeta(z),
$$

where $\Gamma$ is the Euler Gamma function (see $[\mathrm{T},(2.1 .12)]$ ). Then $\xi$ is an entire function of order 1 [T, Theorem 2.12].

For the convenience of the reader, we include the proof of the following proposition.

Proposition 8.1. There exist positive constants $c_{1}<c_{2}, d_{1}<d_{2}$ such that for all $r \geq 2$ we have

$$
\begin{aligned}
& c_{1} r \log r \leq m(r, \zeta) \leq c_{2} r \log r, \\
& d_{1} r \log r \leq m(r, \xi) \leq d_{2} r \log r .
\end{aligned}
$$

Proof. If $x=\operatorname{Re} z>0$, then

$$
\Gamma(z)=\int_{0}^{\infty} t^{z-1} e^{-t} d t, \quad|\Gamma(z)| \leq \Gamma(x) .
$$

We let $\mu(r)$ be the supremum of $\log |\Gamma(z)|$ when $|z|=r$ and $x \geq 1 / 2$. Then by Stirling's formula $C_{1} r \log r \leq \mu(r) \leq C_{2} r \log r$ for $r \geq 2$.

For $x>0$ one has (see [T, (2.1.4)])

$$
\zeta(z)=z \int_{1}^{\infty} \frac{[t]-t+\frac{1}{2}}{t^{z+1}} d t+\frac{1}{z-1}+\frac{1}{2} .
$$

Hence for $x \geq 1 / 2$ and $|z-1|>2$ we have

$$
|\zeta(z)| \leq \frac{|z|}{2} \int_{1}^{\infty} \frac{1}{t^{x+1}} d t+1 \leq|z|+1,
$$

while $\zeta(x)=\sum_{k=1}^{\infty} k^{-x}>1$ for $x>1$. To estimate $\zeta(z)$ for $\boldsymbol{R e} z \leq 1 / 2$ we use the functional equation (see [T, Theorem 2.1])

$$
\zeta(z)=2^{z} \pi^{z-1} \sin \frac{\pi z}{2} \Gamma(1-z) \zeta(1-z) .
$$


We conclude that $m(r, \zeta) \leq c_{2} r \log r$. But for odd integers $n>0$ we have $|\zeta(-n)| \geq 2^{-n} \pi^{-n-1} n$ ! and therefore $m(n, \zeta) \geq c_{1}^{\prime} n \log n$. This implies $m(r, \zeta) \geq c_{1} r \log r, c_{1}>0$.

Using the definition of $\xi(z)$ we have for $|z|=r$ with $\operatorname{Re} z \geq 1 / 2$

$$
|\xi(z)| \leq \frac{r(r+1)^{2}}{2} \Gamma\left(\frac{r}{2}\right), \xi(r) \geq \frac{r(r-1)}{2} \pi^{-r / 2} \Gamma\left(\frac{r}{2}\right) .
$$

Since $\xi(z)=\xi(1-z)$ (see $[\mathrm{T},(2.1 .13)])$ we obtain for $|z|=r$ with $\operatorname{Re} z \leq 1 / 2$

$$
|\xi(z)| \leq \frac{(r+1)(r+2)^{2}}{2} \Gamma\left(\frac{r+1}{2}\right) .
$$

So we see that $d_{1} r \log r \leq m(r, \xi) \leq d_{2} r \log r$.

By Proposition 8.1 the function $\xi$ verifies condition (12) if $k$ is chosen sufficiently large, so Theorem 7.1 and Corollary 7.2 hold for $\xi$. Since $\zeta$ is meromorphic, the quantity $m_{n}(r)$ is not well defined for $\zeta$. We have the following:

Theorem 8.2. There exists a constant $C>0$ such that for every integer $n \geq 1$ and every $r \geq 1$ we have

$$
\frac{n^{2}+3 n}{2} \leq Z_{n}(r, \zeta) \leq C\left(n r \log r+n^{2}\right)
$$

Proof. Since $\zeta$ is holomorphic near 0, we can find, by a simple dimension argument, a non-trivial polynomial $P(z, w)$ of degree at most $n$ such that $P(z, \zeta(z))$ has a zero of order at least $\left(n^{2}+3 n\right) / 2$ at 0 (see the proof of Theorem 2.5 in [CP2]). This implies the lower estimate on $Z_{n}$.

The function $\tilde{\zeta}(z)=(z-1) \zeta(z)$ is entire. Proposition 8.1 implies that

$$
c_{1} r \log r \leq m(r, \tilde{\zeta}) \leq c_{2}^{\prime} r \log r .
$$

By Corollary 7.2 it follows that there exists a constant $C>0$ such that

$$
Z_{n}(r, \tilde{\zeta}) \leq C\left(n r \log r+n^{2}\right),
$$

for all $n \geq 1$ and $r \geq 1$. Note that if $P(z, w)$ is a polynomial of degree at most $n$, then there exists a polynomial $Q(z, w)$ of degree at most $2 n$ such that $(z-1)^{n} P(z, \zeta(z))=Q(z, \tilde{\zeta}(z))$. Hence $Z_{n}(r, \zeta) \leq Z_{2 n}(r, \tilde{\zeta})$, and the proof is complete.

\section{Extremal FunCtions}

If $K \subset \mathbb{C}^{2}$ is a compact set, the extremal function $V_{K}$ of $K$ (also called the pluricomplex Green function of $K$ with pole at infinity) is 
defined by

$$
V_{K}(z, w)=\sup \frac{1}{\operatorname{deg} P} \log |P(z, w)|
$$

where the supremum is taken over all polynomials $P$ such that $\|P\|_{K} \leq$ 1. Then either $V_{K}$ is finite at every point, or $V_{K} \equiv \infty$, and the latter occurs if and only if $K$ is pluripolar (see e.g. [K, Ch. 5]).

Let $f$ be an entire transcendental function and let

$$
K=\{(z, f(z)):|z| \leq 1\}
$$

Then $K$ is pluripolar and $V_{K} \equiv \infty$. Using our estimates on $m_{n}(r)$, it is still possible to define a meaningful extremal function of $K$ along the graph of $f$. This relates to Sadullaev's result on the existence of extremal functions for non-pluripolar subsets of algebraic varieties [Sa].

We assume in this section that $f$ is an entire transcendental function which verifies

$$
m_{n}(r) \leq C_{f} n^{2} \log r, 1 \leq r \leq r_{n}, n \geq 1,
$$

where $r_{n}$ is a sequence increasing to infinity and $C_{f}$ is a constant depending on $f$. Classes of such functions are constructed in Section 7 . Let us define

$$
W_{n}(z)=\sup \log |P(z, f(z))|,
$$

where the supremum is taken over all polynomials $P$ of degree at most $n$ which verify $|P(z, f(z))| \leq 1$ on $\Delta$. The functions $W_{n}$ are non-negative, continuous and subharmonic on $\mathbb{C}$, and $W_{n} \equiv 0$ on $\Delta$.

Next we define

$$
W(z)=\limsup _{n \rightarrow \infty} \frac{1}{n^{2}} W_{n}(z)
$$

and we let $W^{\star}$ denote the upper semicontinuous regularization of $W$. We have the following:

Proposition 9.1. The function $W^{\star}$ is non-negative subharmonic on $\mathbb{C}, W^{\star} \equiv 0$ on $\Delta$, and for all $r \geq 1$

$$
\frac{1}{2} \log r \leq \max \left\{W^{\star}(z):|z|=r\right\} \leq C_{f} \log r .
$$

If $f(z)=e^{z}$ then $W^{\star}(z)=\frac{1}{2} \log ^{+}|z|$ for all $z \in \mathbb{C}$.

Proof. By the proofs of Theorem 2.5 and Corollary 2.6 of [CP2] there exists, for each $n \geq 1$, a non-trivial polynomial $P_{n}(z, w)$ of degree $n$, such that the function $F_{n}(z)=P_{n}(z, f(z))$ verifies $M\left(1, F_{n}\right)=1$ and

$$
\frac{n^{2}+3 n}{2} \log r \leq \log M\left(r, F_{n}\right) \leq m_{n}(r),
$$

for all $r \geq 1$. Note that, in particular, this implies $C_{f} \geq 1 / 2$ for any $f$. 
Our assumption on the growth of $m_{n}(r)$ implies that the family of subharmonic functions $W_{n} / n^{2}$ is locally upper bounded, hence $W^{\star}$ is a non-negative subharmonic function on $\mathbb{C}$ which verifies

$$
W^{\star}(z) \leq C_{f} \log ^{+}|z| .
$$

Suppose that for some $r>1$ we have

$$
\max \left\{W^{\star}(z):|z|=r\right\}<\frac{1}{2} \log r .
$$

The Hartogs Lemma implies that for $n$ large and for all $z$ with $|z|=r$

$$
\log \left|F_{n}(z)\right| \leq W_{n}(z)<\frac{n^{2}}{2} \log r .
$$

This contradicts the above lower estimate on $\log M\left(r, F_{n}\right)$.

In the case of the function $f(z)=e^{z}$ it was proved in [CP1] that

$$
m_{n}(r) \leq\left(\frac{n^{2}}{2}+o\left(n^{2}\right)\right) \log r, 1 \leq r \leq n .
$$

The preceding argument shows that now $W^{\star}(z) \leq \frac{1}{2} \log ^{+}|z|$. We conclude that the equality must hold, by applying the maximum principle on $\mathbb{C} \backslash \Delta$ to the subharmonic function $W^{\star}(z)-\frac{1}{2} \log |z| \leq 0$.

\section{Estimates for Algebraic MeAsures}

Throughout Sections 10 and $11, K$ is an algebraic extension of degree $\sigma$ of the field $\mathbb{Q}$ of rational numbers and $f$ is, unless otherwise specified, an entire transcendental function of finite positive order $\rho$. Without loss of generality we may assume that $M(r, f) \geq r$ for $r \geq 1$.

For an algebraic number $\zeta$, we define its norm $\|\zeta\|$ as the maximum of the absolute values of its conjugates. Then $\left\|\zeta_{1} \zeta_{2}\right\| \leq\left\|\zeta_{1}\right\|\left\|\zeta_{2}\right\|$ and $\left\|\zeta_{1}+\zeta_{2}\right\| \leq\left\|\zeta_{1}\right\|+\left\|\zeta_{2}\right\|$ (see $[\mathrm{M}$, p. 62$\left.]\right)$.

If $P\left(\zeta_{1}, \ldots, \zeta_{n}\right)$ is a polynomial with algebraic coefficients, then its height $h(P)$ is defined as the maximum of the norms of its coefficients.

If $\omega_{1}, \ldots, \omega_{\sigma}$ is a basis for the ring $I_{K}$ of algebraic integers in $K$, then any $\zeta \in I_{K}$ can be written as

$$
\zeta=p_{1} \omega_{1}+\cdots+p_{\sigma} \omega_{\sigma}
$$

where $p_{1}, \ldots, p_{\sigma}$ are rational integers. If ||$|\zeta|||=\max \left\{\left|p_{1}\right|, \ldots,\left|p_{\sigma}\right|\right\}$, then (see [M, p. 62]) there are constants $\gamma_{1}$ and $\gamma_{2}$ depending only on $K$ such that $\gamma_{1}|\|\zeta\||\left|\leq\|\zeta\| \leq \gamma_{2}\right|\|\zeta\| \mid$.

Given a natural number $d$ we denote by $I_{K}(d)$ the set of numbers $z \in K$ such that $d z \in I_{K}$, and by $I_{K}(d, A)$ the set of $z \in I_{K}(d)$ with $\|z\| \leq A$. Let $N_{K}(d, A, r)$ be the number of points in $I_{K}(d, A) \cap \Delta_{r}$. 
Lemma 10.1. There exist constants $c^{\prime}$ and $c^{\prime \prime}$ depending only on $K$ with the following properties: If $K$ is real and $r \leq A$ then

$$
c^{\prime} d^{\sigma} A^{\sigma-1} r \leq N_{K}(d, A, r) \leq c^{\prime \prime} d^{\sigma} A^{\sigma-1} r .
$$

If $K$ is complex and $r \leq A$ then

$$
c^{\prime} d^{\sigma} A^{\sigma-2} r^{2} \leq N_{K}(d, A, r) \leq c^{\prime \prime} d^{\sigma} A^{\sigma-2} r^{2} .
$$

Moreover, in both cases, if $r>A$ then

$$
c^{\prime}(d A)^{\sigma} \leq N_{K}(d, A, r) \leq c^{\prime \prime}(d A)^{\sigma} .
$$

Proof. We will consider only the complex case, so $\sigma \geq 2$. The real case can be considered in a similar manner.

We assume at first that $d=1$. If $N$ is the number of points in $I_{K}(1, A)$, then by $(13) c_{1} A^{\sigma} \leq N \leq c_{2} A^{\sigma}$, where the constants $c_{1}$ and $c_{2}$ depend only on $K$. Moreover $I_{K}(1, A) \subset \Delta_{A}$, so the lemma is proved for $r>A$.

Suppose that $r \leq A$. There exists an absolute constant $c_{3}$ such that $\Delta_{A}$ can be covered by $c_{3} A^{2} r^{-2}$ disks of radius $r / 2$. Therefore there is a disk $D$ of radius $r / 2$ containing at least $c_{4} A^{\sigma-2} r^{2}$ points of $I_{K}(1, A / 2)$, where $c_{4}$ depends only on $K$. Let $z_{0}$ be one of this points. If $z \in I_{K}(1, A / 2) \cap D$, then $z-z_{0} \in I_{K}(1, A) \cap \Delta_{r}$. Consequently, $N_{K}(1, A, r) \geq c^{\prime} A^{\sigma-2} r^{2}$, where $c^{\prime}$ depends only on $K$.

Now let $N=N_{K}(1, A, r)$ and let $\omega_{1}, \ldots, \omega_{\sigma}$ be a basis for $I_{K}$ over $\mathbb{Z}$. Since $I_{K} \not \subset \mathbb{R}$, we may assume that $\omega_{1} / \omega_{2} \notin \mathbb{R}$. Then it is easy to see that there is a constant $c_{5}$ depending only on $K$ such that the disk $\Delta_{A}$ contains at least $c_{5} A^{2}$ points $z$ of the form $z=p_{1} \omega_{1}+p_{2} \omega_{2}$, where $p_{1}, p_{2} \in \mathbb{Z}$ and $\left|p_{1}\right|,\left|p_{2}\right| \leq A / \gamma_{2}$, so $\|z\| \leq A$. Moreover, there is a constant $c_{6}$ depending only on $K$ and at least $c_{6} A^{2} r^{-2}$ disjoint disks of radius $r$ centered at these points. Hence each of these disks contains at least $N$ points from $I_{K}(1,2 A)$. It follows that $c_{6} N A^{2} r^{-2} \leq c_{2} 2^{\sigma} A^{\sigma}$, so $N \leq c^{\prime \prime} A^{\sigma-2} r^{2}$.

If $d>1$ then we note that $z \in I_{K}(1, d A, d r)$ if and only if $z / d \in$ $I_{K}(d, A, r)$, hence $N_{K}(d, A, r)=N_{K}(1, d A, d r)$.

We say that a function $f$ takes values at $z$ in $I_{K}(d)$ with multiplicity $m$ if the numbers $z, f(z), \ldots, f^{(m-1)}(z)$ belong to $I_{K}(d)$. In this case we define $\|f(z)\|_{m}$ as the maximum of $\|z\|,\|f(z)\|, \ldots,\left\|f^{(m-1)}(z)\right\|$.

In this setting, we have the following lemma (see Ch. 1, $\S 2$ and Ch. $2, \S 2$ in $[\mathrm{G}])$.

Lemma 10.2. Let $f$ be a holomorphic function in a neighborhood of $z_{0}$, which takes values at $z_{0}$ in $I_{K}(d)$ with multiplicity $m$, and such that $\left\|f\left(z_{0}\right)\right\|_{m} \leq A, A \geq 1$. If $P(z, w)$ is a polynomial of degree $n$ with 
coefficients in $I_{K}$ and of height $h$, and if $F(z)=P(z, f(z))$, then for $k \leq m-1$ we have $F^{(k)}\left(z_{0}\right) \in I_{K}\left(d^{n}\right)$ and

$$
\left\|g_{i j}^{(k)}\left(z_{0}\right)\right\| \leq A^{i+j}(i+j)^{k},
$$

where $g_{i j}(z)=z^{i} f^{j}(z), i+j \leq n$. Moreover, if $F^{(k)}\left(z_{0}\right) \neq 0$ then

$$
\left|d^{n} F^{(k)}\left(z_{0}\right)\right| \geq\left(h d^{n} A^{n}(n+1)^{k+2}\right)^{-\sigma+1} .
$$

Proof. If

$$
f(z)=\sum_{j=0}^{\infty} a_{j}\left(z-z_{0}\right)^{j},
$$

then the $k$-th derivative $a_{j k}$ of $f^{j}(z)$ at $z_{0}$ is

$$
a_{j k}=k ! \sum_{i_{1}+\cdots+i_{j}=k} a_{i_{1}} \cdots a_{i_{j}}=k ! \sum_{i_{1}+\cdots+i_{j}=k} \frac{f^{\left(i_{1}\right)}\left(z_{0}\right) \cdots f^{\left(i_{j}\right)}\left(z_{0}\right)}{i_{1} ! \cdots i_{j} !} .
$$

Hence $d^{j} a_{j k} \in I_{K}$. Since

$$
j^{k}=\sum_{i_{1}+\cdots+i_{j}=k} \frac{k !}{i_{1} ! \cdots i_{j} !},
$$

we see that $\left\|a_{j k}\right\| \leq j^{k} A^{j}$.

If $g_{i j}(z)=z^{i} f^{j}(z)$, then

$$
g_{i j}^{(k)}\left(z_{0}\right)=\sum_{p=0}^{\min \{i, k\}}\left(\begin{array}{l}
k \\
p
\end{array}\right) \frac{i !}{(i-p) !} z_{0}^{i-p} a_{j, k-p} .
$$

Thus $d^{i+j} g_{i j}^{(k)}\left(z_{0}\right) \in I_{K}$. Moreover,

$$
\begin{aligned}
\left\|g_{i j}^{(k)}\left(z_{0}\right)\right\| & \leq \sum_{p=0}^{\min \{i, k\}}\left(\begin{array}{l}
k \\
p
\end{array}\right) \frac{i !}{(i-p) !} j^{k-p} A^{i+j-p} \\
& \leq A^{i+j} \sum_{p=0}^{k}\left(\begin{array}{l}
k \\
p
\end{array}\right) i^{p} j^{k-p}=A^{i+j}(i+j)^{k} .
\end{aligned}
$$

Hence $d^{n} F^{(k)}\left(z_{0}\right) \in I_{K}$ and

$$
\left\|F^{(k)}\left(z_{0}\right)\right\| \leq \frac{(n+1)(n+2)}{2} h A^{n} n^{k} \leq h A^{n}(n+1)^{k+2} .
$$

Since $c_{1}=d^{n} F^{(k)}\left(z_{0}\right) \in I_{K}$, the number $\nu$ of its conjugates $c_{2}, \ldots, c_{\nu}$ does not exceed $\sigma$ and

$$
\left|\prod_{i=1}^{\nu} c_{i}\right| \geq 1
$$


when $c_{1} \neq 0$. Note that $\left|c_{i}\right| \leq\left\|c_{i}\right\|=\left\|c_{1}\right\|$. Consequently,

$$
\left|c_{1}\right| \geq\left(h d^{n} A^{n}(n+1)^{k+2}\right)^{-\sigma+1} \text {. }
$$

The following result is a consequence of C. L. Siegel's lemma adapted for our purposes.

Lemma 10.3. Suppose that there are l points $z_{1}, \ldots, z_{l}$ in $\Delta_{r}, r \geq 1$, such that, for $1 \leq q \leq l$, $f$ takes values at $z_{q}$ in $I_{K}\left(d_{q}\right)$ with multiplicity $m_{q}$ and $\left\|f\left(z_{q}\right)\right\|_{m_{q}} \leq A, A \geq 1$. If $\nu=\sum_{q=1}^{l} m_{q}<N$, where $N=$ $(n+1)(n+2) / 2$, and $m_{q} \leq m, d_{q} \leq d$, then there is a polynomial $P(z, w)$ of degree $n$ with coefficients $c_{i j} \in I_{K}$ and of height

$$
h(P) \leq H_{n}=C_{1}\left(C_{2} d^{n} A^{n}(n+1)^{m+1}\right)^{\nu /(N-\nu)},
$$

where $C_{1}, C_{2}$ are constants depending only on $K$, with the following properties: The function

$$
F(z)=P(z, f(z))=\sum_{i+j=0}^{n} c_{i j} z^{i} f^{j}(z) \not \equiv 0
$$

and for $t \geq 2 r$

$$
\|F\|_{\Delta_{2 r}} \leq(n+1)^{2} H_{n}\left(\frac{4 r}{t}\right)^{\mu} M^{n}(t, f),
$$

where $\mu \geq \nu$ is the number of zeros of $F$ in $\Delta_{r}$.

Proof. By Lemma $10.2 d_{q}^{n} g_{i j}^{(k)}\left(z_{q}\right)$, where $g_{i j}(z)=z^{i} f^{j}(z)$, is an algebraic integer and

$$
\left\|d_{q}^{n} g_{i j}^{(k)}\left(z_{q}\right)\right\| \leq d_{q}^{n} A^{n} n^{k} \leq d^{n} A^{n} n^{m-1} .
$$

Let us consider the system of $\nu$ equations

$$
\sum_{i+j=0}^{n} c_{i j} d_{q}^{n} g_{i j}^{(k)}\left(z_{q}\right)=0, \quad 1 \leq q \leq l, \quad 0 \leq k \leq m_{q}-1,
$$

with $N$ unknowns $c_{i j}$. By $\left[\mathrm{M}, \mathrm{p}\right.$. 63] there are constants $C_{1}$ and $C_{2}$ depending only on $K$ such that this system has a non-trivial solution in $I_{K}$ with

$$
\left\|c_{i j}\right\| \leq C_{1}\left(C_{2} N d^{n} A^{n} n^{m-1}\right)^{\nu /(N-\nu)} \leq H_{n} .
$$

Since $\|P\|_{\Delta^{2}} \leq(n+1)^{2} H_{n}$, by the Bernstein-Walsh inequality

$$
|P(z, w)| \leq(n+1)^{2} H_{n} \exp \left(n \max \left\{\log ^{+}|z|, \log ^{+}|w|\right\}\right),
$$

so $\|F\|_{\Delta_{t}} \leq(n+1)^{2} H_{n} M^{n}(t, f)$. 
The function $F$ has $\mu \geq \nu$ zeros in $\Delta_{r}$, so by Lemma 6.1

$$
\|F\|_{\Delta_{2 r}} \leq\left(\frac{4 r}{t}\right)^{\mu}\|F\|_{\Delta_{t}} \leq(n+1)^{2} H_{n}\left(\frac{4 r}{t}\right)^{\mu} M^{n}(t, f) .
$$

Suppose that for a set $E \subset \mathbb{C}$ and for some integer $m \geq 1$ we have $z, f(z), \ldots, f^{(m-1)}(z) \in K$ for all $z \in E$. Then for $z \in E$ we let $d_{z}$ be the smallest natural number such that $f$ takes values at $z$ in $I_{K}\left(d_{z}\right)$ with multiplicity $m$. We set

$$
\begin{gathered}
\|f\|_{E, m}=\max \left\{1, \sup _{z \in E}\|f(z)\|_{m}\right\}, d(E, m)=\sup _{z \in E} d_{z}, \\
\mathcal{A}_{K}(E, m)=d(E, m)\|f\|_{E, m} .
\end{gathered}
$$

The number $\mathcal{A}_{K}(E, m)$ will be called the algebraic measure of order $m$ of the function $f$ on $E$. If for some $z \in E$ we have $z \notin K$ or $f^{(j)}(z) \notin K$ for some $j<m$, then we set $\mathcal{A}_{K}(E, m)=\infty$. Note also that if a set $E$ is infinite then $\mathcal{A}_{K}(E, m)=\infty$ for every $m \geq 1$.

Throughout the rest of this section and in Section 11 we will assume that $m(r) \leq r^{\phi(r)}$, where $\lim _{r \rightarrow \infty} \phi(r)=\rho$ and the function $r^{\phi(r)-\rho}$ is slowly increasing. We denote by $r_{n}$ the unique solution of the equation $r^{\phi(r)}=n$. The following result is the main tool in the forthcoming estimates of the algebraic measure.

Theorem 10.4. There exists a constant $C_{K}$ depending only on $K$ with the following property: If $n \geq 1,1 \leq r \leq r_{n} / 4$ and $E \subset \Delta_{r}$, then there are integers $k \geq 0$ and $\mu$ such that

$$
\begin{aligned}
& (k+1)|E|>n^{2} / 4, \max \left\{n^{2} / 4, k|E|\right\} \leq \mu \leq Z_{n}(r), \\
& C_{K} \mathcal{A}_{K}^{2 \sigma}(E, k+1) \geq\left(\frac{r}{k(n+1)^{2 \sigma-1}}\right)^{k / n} \exp \left(\frac{\mu}{n} \log \frac{r_{n}}{4 e^{4} r}\right) .
\end{aligned}
$$

In the above statement we let $k^{k}=1$ if $k=0$.

Proof. We may assume that $E$ is finite. Let $E=\left\{z_{1}, \ldots, z_{l}\right\}$ and $\nu=\left[n^{2} / 4\right]+1$. Note that $\nu /(N-\nu) \leq 1$ and by Theorem 2.5 and Corollary 2.6 in [CP2] we have $\nu \leq\left(n^{2}+3 n\right) / 2 \leq Z_{n}(r)$.

Let $m=[\nu / l]$. If $\mathcal{A}_{K}(E, m+1)=\infty$, then we take $k=m$ and $\mu=\nu$ and the proof is finished. Otherwise, we let $A_{1}=\|f\|_{E, m+1}$ and $d_{1}=d(E, m+1)$. We have $\nu=m l+p, 0 \leq p \leq l-1$. Applying Lemma 10.3 with the above points, with $m_{q}=m+1$ when $1 \leq q \leq p$ and $m_{q}=m$ when $p+1 \leq q \leq l$, and with this value of $\nu$, we construct a non-trivial polynomial $P(z, w)$ of degree $n$ with coefficients in $I_{K}$ and with height

$$
h(P) \leq h=\underset{33}{C_{1} C_{2} d_{1}^{n} A_{1}^{n}(n+1)^{m+2}}
$$


such that

$$
\|F\|_{\Delta_{2 r}} \leq(n+1)^{2} h\left(\frac{4 r}{t}\right)^{\mu} M^{n}(t, f),
$$

where $\mu \geq n^{2} / 4$ is the number of zeros of $F$ in $\Delta_{r}$ and $t \geq 2 r$.

There exist $q$ and $k, 1 \leq q \leq l, 0 \leq k \leq \mu / l$, such that $F^{(k)}\left(z_{q}\right) \neq 0$. We may assume that $\mathcal{A}_{K}(E, k+1)<\infty$. Clearly, $k \geq m$ and therefore $k l>\nu-l>n^{2} / 4-l$. Moreover $A=\|f\|_{E, k+1} \geq A_{1}, d=d(E, k+1) \geq$ $d_{1}$, so

By Lemma 10.2

$$
h \leq C_{1} C_{2} d^{n} A^{n}(n+1)^{k+2} .
$$

$$
\begin{aligned}
\left|d^{n} F^{(k)}\left(z_{q}\right)\right| & \geq\left(h d^{n} A^{n}(n+1)^{k+2}\right)^{-\sigma+1} \\
& \geq\left(C_{1} C_{2} d^{2 n} A^{2 n}(n+1)^{2 k+4}\right)^{-\sigma+1} .
\end{aligned}
$$

By Cauchy's inequalities

$$
\begin{aligned}
\left|d^{n} F^{(k)}\left(z_{q}\right)\right| & \leq d^{n} k ! \frac{\|F\|_{\Delta_{2 r}}}{r^{k}} \leq d^{n}(n+1)^{2} h\left(\frac{k}{r}\right)^{k}\left(\frac{4 r}{t}\right)^{\mu} M^{n}(t, f) \\
& \leq C_{1} C_{2} d^{2 n} A^{n}(n+1)^{k+4}\left(\frac{k}{r}\right)^{k}\left(\frac{4 r}{t}\right)^{\mu} M^{n}(t, f) .
\end{aligned}
$$

We obtain

$$
\begin{aligned}
& \left(C_{1} C_{2} d^{2 n} A^{2 n}(n+1)^{2 k+4}\right)^{-\sigma+1} \leq \\
& C_{1} C_{2} d^{2 n} A^{n}(n+1)^{k+4}\left(\frac{k}{r}\right)^{k}\left(\frac{4 r}{t}\right)^{\mu} M^{n}(t, f) .
\end{aligned}
$$

Since $A \geq 1$ this implies

$$
\left(C_{1} C_{2}\right)^{\sigma}\left(d^{2 n} A^{2 n}\right)^{\sigma} \geq\left(\frac{r}{k}\right)^{k}\left(\frac{t}{4 r}\right)^{\mu} M^{-n}(t, f)(n+1)^{-k(2 \sigma-1)-4 \sigma} .
$$

Let $C_{K}=\left(16 C_{1} C_{2}\right)^{\sigma}$. Taking the $n$th root and using the inequality $(n+1)^{-1 / n} \geq 1 / 2$, we get

$$
C_{K} \mathcal{A}^{2 \sigma}(E, k+1) \geq\left(\frac{r}{k(n+1)^{2 \sigma-1}}\right)^{k / n}\left(\frac{t}{4 r}\right)^{\mu / n} M^{-1}(t, f) .
$$

Let $t=r_{n}$. Since $\mu \geq n^{2} / 4,4 r \leq r_{n}$ and $M\left(r_{n}, f\right) \leq e^{n}$, we get

so

$$
\left(\frac{r_{n}}{4 r}\right)^{\mu / n} M^{-1}\left(r_{n}, f\right) \geq\left(\frac{r_{n}}{4 r}\right)^{\mu / n} e^{-4 \mu / n}=\exp \left(\frac{\mu}{n} \log \frac{r_{n}}{4 e^{4} r}\right),
$$

$$
C_{K} \mathcal{A}_{K}^{2 \sigma}(E, k+1) \geq\left(\frac{r}{k(n+1)^{2 \sigma-1}}\right)^{k / n} \exp \left(\frac{\mu}{n} \log \frac{r_{n}}{4 e^{4} r}\right) .
$$




\section{Algebraic Growth of transcendental FunCtions}

Let $f$ be an entire function of finite order $\rho$ and $K$ be an algebraic number field of degree $\sigma=[K: \mathbb{Q}]$. As in Section 10 we assume, without loss of generality, that $m(r) \leq r^{\phi(r)}$, where $\phi(r) \rightarrow \rho$ and $r^{\phi(r)-\rho}$ is a slowly increasing function. Recall the definition of the sequence $\left\{r_{n}\right\}$ by the equations $r_{n}^{\phi\left(r_{n}\right)}=n$.

Given a transcendental function $f$ we define the algebraic growth characteristic of $f$ on $K$ by

$$
\mathbf{a}_{K}(s, r, m)=\inf \left\{\log \mathcal{A}_{K}(E, m): E \subset \Delta_{r},|E| \geq s\right\} .
$$

Due to our knowledge of the behavior of $Z_{n}(r)$ we are now able to get estimates for $\mathbf{a}_{K}(s, r, m)$. The first series of results applies to general transcendental functions. We recall that when $\sigma>2$, then for every $\epsilon>0$ there is an entire function $f$ of order smaller than $\epsilon$ such that $f(K) \subset I_{K}$ (see [GS, Satz 1]). Moreover, one can find such a function so that $f^{(m)}(K) \subset K$ for all $m$ (see [GS, Satz 2]) .

Our first theorem shows that when $m$ and $r$ are fixed, the algebraic growth characteristic exceeds $s^{1 / 2} \log s$, at least for a subsequence of integers $s$.

Theorem 11.1. If $f$ has finite order $\rho>0$ then for all $m, r \geq 1$

$$
\limsup _{s \rightarrow \infty} \frac{\mathbf{a}_{K}(s, r, m)}{s^{1 / 2} \log s}>\frac{2^{-3 \rho / 2-10}}{\sigma}\left(\frac{\Lambda m}{\rho(\rho+5)}\right)^{1 / 2} .
$$

Proof. By Corollary 6.4 there is a fundamental sequence of integers $\left\{n_{j}\right\}$ for $f$ with the following property: For every $r \geq 1$ there is an integer $j_{r}$ such that $Z_{n_{j}}(r) \leq a n_{j}^{2}$ for $j \geq j_{r}$, where

$$
a=\frac{2^{3 \rho+11}(\rho+5)}{\Lambda \rho} .
$$

We may assume that $4 r \leq r_{n_{j}}$ when $j \geq j_{r}$. Let $s_{j}=a n_{j}^{2} / m+1$ and $E$ be a subset of $\Delta_{r}$ with $|E| \geq s_{j}$. If $k$ is the integer from Theorem 10.4 corresponding to $n=n_{j}, r, E$, then $m|E|>Z_{n_{j}}(r) \geq k|E|$, so $m \geq k+1$. It follows from Theorem 10.4 that

$$
C_{K} \mathcal{A}_{K}^{2 \sigma}(E, m) \geq\left(m\left(n_{j}+1\right)^{2 \sigma-1}\right)^{-m / n_{j}} \exp \left(\frac{n_{j}}{4} \log \frac{r_{n_{j}}}{4 e^{4} r}\right) .
$$

For all $j$ sufficiently large (depending on $r, m$ ) we have

$$
\left(m\left(n_{j}+1\right)_{35}^{2 \sigma-1}\right)^{-m / n_{j}} \geq 1 / 2 .
$$


Since $r_{n_{j}}^{\phi\left(r_{n_{j}}\right)}=n_{j}$ and $\phi\left(r_{n_{j}}\right) \rightarrow \rho$, we conclude that there is a sequence of positive $\epsilon_{j} \rightarrow 0$ such that

$$
\frac{n_{j}}{4} \log \frac{r_{n_{j}}}{4 e^{4} r} \geq \frac{\left(1-\epsilon_{j}\right) n_{j}}{4 \rho} \log n_{j} .
$$

It follows that

$$
2 \sigma \mathbf{a}_{K}\left(s_{j}, r, m\right)+\log \left(2 C_{K}\right) \geq \frac{\left(1-\epsilon_{j}\right) n_{j}}{4 \rho} \log n_{j} .
$$

Since $n_{j}=\left(m\left(s_{j}-1\right) / a\right)^{1 / 2}$ we see that

$$
\limsup _{s \rightarrow \infty} \frac{\mathbf{a}_{K}(s, r, m)}{s^{1 / 2} \log s} \geq \frac{(m / a)^{1 / 2}}{16 \sigma \rho}>\frac{2^{-3 \rho / 2-10}}{\sigma}\left(\frac{\Lambda m}{\rho(\rho+5)}\right)^{1 / 2} .
$$

Remark: It is interesting to note that the value of lim sup in the above theorem is achieved on a sequence $\left\{s_{j}\right\}$ depending only on $f$ and $m$, $s_{j}=a n_{j}^{2} / m+1$.

As mentioned above, there are functions whose derivatives of all orders map $K$ into $K$. So it is interesting to estimate the algebraic growth of such functions on the sets $I_{K}(d, A)$. The number $d A$ can be viewed as the algebraic measure of the set $I_{K}(d, A)$, while $\mathcal{A}_{K}\left(I_{K}(d, A), m\right)$ is the algebraic measure of the set of values of $f$ and its derivatives on $I_{K}(d, A)$. We introduce

$$
\eta_{K}(\lambda, r, m)=\inf \left\{\log \mathcal{A}_{K}\left(I_{K}(d, A) \cap \Delta_{r}, m\right): d A \geq \lambda\right\} .
$$

The following result describes the growth of $\eta_{K}(\lambda, r, m)$.

Corollary 11.2. If $\sigma \geq 3$, then there is a constant $c^{\prime}$ depending only on $K$ such that for $r, m \geq 1$

$$
\limsup _{\lambda \rightarrow \infty} \frac{\eta(\lambda, r, m)}{\lambda^{\sigma / 2-1} \log \lambda} \geq \frac{\sigma-2}{\sigma} 2^{-3 \rho / 2-10}\left(\frac{c^{\prime} \Lambda m}{\rho(\rho+5)}\right)^{1 / 2} .
$$

Proof. By Lemma 10.1, $\left|I_{K}(d, A) \cap \Delta_{r}\right| \geq c^{\prime}(d A)^{\sigma-2}$. By Theorem 11.1, let $s_{j}$ be a sequence such that

$$
\limsup _{j \rightarrow \infty} \frac{\mathbf{a}_{K}\left(s_{j}, r, m\right)}{s_{j}^{1 / 2} \log s_{j}} \geq \frac{2^{-3 \rho / 2-10}}{\sigma}\left(\frac{\Lambda m}{\rho(\rho+5)}\right)^{1 / 2} .
$$

We define $\lambda_{j}$ by $s_{j}=c^{\prime} \lambda_{j}^{\sigma-2}$. If $d A \geq \lambda_{j}$ then $\eta\left(\lambda_{j}, r, m\right) \geq \mathbf{a}_{K}\left(s_{j}, r, m\right)$, SO

$$
\limsup _{j \rightarrow \infty} \frac{\eta\left(\lambda_{j}, r, m\right)}{\lambda_{j}^{\sigma / 2-1} \log \lambda_{j}} \geq \sqrt{c^{\prime}}(\sigma-2) \limsup _{j \rightarrow \infty} \frac{\mathbf{a}_{K}\left(s_{j}, r, m\right)}{s_{j}^{1 / 2} \log s_{j}},
$$


and the conclusion follows.

Let $I_{K}(A)=I_{K}(1, A)$ be the set of algebraic integers $z \in I_{K}$ of norm $\|z\| \leq A$. Clearly, $I_{K}(A) \subset \Delta_{A}$. In our next theorem we estimate the number of points $z \in I_{K}(A)$ which are mapped to points of $I_{K}$ of smallest possible norm $A^{\prime}$. Since $|z| \leq A$ and $|f(z)| \leq\|f(z)\|$, it is natural to expect, due to the growth of $f$, that $A^{\prime} \geq \exp \left(A^{\phi(A)}\right)$. We will prove that if $\rho<\sigma / 2$ then the proportion of points of $I_{K}\left(A_{j}\right)$ which are mapped by $f$ into $I_{K}\left(\exp A_{j}^{\phi\left(A_{j}\right)}\right)$ tends to 0 , for a certain sequence $A_{j} \rightarrow \infty$.

To this end, we need the following version of Theorem 10.4, which provides upper bounds for $|E|$ if the algebraic measure of order 1 of $f$ on $E$ is bounded above by certain quantities.

Proposition 11.3. There exists a constant $C_{K}$ depending only on $K$ such that if $n \geq 1,1 \leq r \leq r_{n} / 4, E \subset \Delta_{r}$ and

$$
C_{K} \mathcal{A}_{K}^{2 \sigma}(E, 1)<\exp \left(\frac{n}{4} \log \frac{r_{n}}{4 e^{4} r}\right),
$$

then $|E| \leq Z_{n}(r)$.

Proof. If $|E|>Z_{n}(r)$ and $k$ is the integer from Theorem 10.4, then $k|E| \leq Z_{n}(r)$ implies $k=0$. Since $\mu \geq n^{2} / 4$, we reach a contradiction with the conclusion of Theorem 10.4.

We have the following theorem:

Theorem 11.4. If $f$ is an entire function of order $0<\rho<\sigma / 2$ then

$$
\liminf _{A \rightarrow \infty} \frac{\left|I_{K}(A) \cap f^{-1}\left(I_{K}\left(\exp A^{\phi(A)}\right)\right)\right|}{\left|I_{K}(A)\right|}=0 .
$$

Proof. By Theorem 5.4 and Corollary 2.6 in [CP2], there exists a fundamental sequence $\left\{n_{j}\right\}$ for $f$ and positive numbers $\epsilon_{j} \rightarrow 0$ such that

$$
Z_{n_{j}}(r) \leq a n_{j}^{2} \log 3 r, 1 \leq r \leq n_{j}^{1 / \rho-\epsilon_{j}} / 6,
$$

where $a=2^{3 \rho+10}(\rho+5) /(\Lambda \rho)$.

Let $A_{j}=n_{j}^{1 /((1+\epsilon) \rho)}$, where $\epsilon>0$ is chosen so that $\sigma>2(1+\epsilon) \rho$, and let $E_{j}=I_{K}\left(A_{j}\right) \cap f^{-1}\left(I_{K}\left(\exp A_{j}^{\phi\left(A_{j}\right)}\right)\right)$. Then

$$
\mathcal{A}_{K}\left(E_{j}, 1\right) \leq \exp A_{j}^{\phi\left(A_{j}\right)}=\exp n_{j}^{\phi\left(A_{j}\right) /((1+\epsilon) \rho)},
$$

and for $j$ sufficiently large

$$
Z_{n_{j}}\left(A_{j}\right) \leq a n_{j}^{2} \log 3 A_{j}=a A_{j}^{2(1+\epsilon) \rho} \log 3 A_{j} .
$$


Since $r_{n_{j}}=n_{j}^{1 / \phi\left(r_{n_{j}}\right)}$ and $\phi\left(r_{n_{j}}\right) \rightarrow \rho$, we have $A_{j}<r_{n_{j}} /\left(8 e^{4}\right)$ for all $j$ sufficiently large, hence

$$
\frac{n_{j}}{4} \log \frac{r_{n_{j}}}{4 e^{4} A_{j}}>\frac{n_{j}}{4} \log 2
$$

As $\phi\left(A_{j}\right) /((1+\epsilon) \rho) \rightarrow 1 /(1+\epsilon)$ as $j \rightarrow \infty$, we conclude that for all $j$ sufficiently large we have

$$
C_{K} \mathcal{A}_{K}^{2 \sigma}\left(E_{j}, 1\right)<\exp \left(\frac{n_{j}}{4} \log \frac{r_{n_{j}}}{4 e^{4} A_{j}}\right) .
$$

Proposition 11.3 implies that $\left|E_{j}\right| \leq Z_{n_{j}}\left(A_{j}\right)$, so

$$
A_{j}^{-\sigma}\left|E_{j}\right| \leq a A_{j}^{2(1+\epsilon) \rho-\sigma} \log 3 A_{j} \rightarrow 0
$$

as $j \rightarrow \infty$. The theorem follows by Lemma 10.1, as $\left|I_{K}\left(A_{j}\right)\right| \geq c^{\prime} A_{j}^{\sigma}$, with a constant $c^{\prime}$ depending only on $K$.

The theorems of Polya and Gelfond state that if an entire transcendental function takes integer values at all integer points, or Gaussian integer values at all Gaussian integer points, then its order is at least 1, and respectively at least 2 . Using Theorem 11.4, we can obtain asymptotic estimates for the number of integer (or Gaussian integer) points in the disk of radius $A$, where a function $f$ takes integer (respectively Gaussian integer) values.

Corollary 11.5. Let $K$ be either $\mathbb{Q}$ or $\mathbb{Q}(i \sqrt{p})$, where $p>0$ is a square free integer. If $f$ is an entire function of order $0<\rho<\sigma / 2$ then

$$
\liminf _{A \rightarrow \infty} \frac{\left|I_{K} \cap f^{-1}\left(I_{K}\right) \cap \Delta_{A}\right|}{\left|I_{K} \cap \Delta_{A}\right|}=0 .
$$

Proof. Note that for $z \in K$ we have $\|z\|=|z|$, so

$$
I_{K} \cap \Delta_{A}=I_{K}(A), I_{K} \cap f^{-1}\left(I_{K}\right) \cap \Delta_{A}=I_{K}(A) \cap f^{-1}\left(I_{K}\left(\exp A^{\phi(A)}\right)\right),
$$

for every $A>0$. The conclusion now follows from Theorem 11.4.

We conclude by considering entire transcendental functions which have a covering system of admissible intervals $I\left(R_{j}, \alpha, \beta, \gamma, C\right.$ ) (see Corollary 4.3). Classes of such functions were constructed in Section 7. In this case we can estimate $\mathbf{a}_{K}(s, r, m)$ for fixed values of $r, m$ and for all $s$ sufficiently large. Let $\tau=1+1 / \gamma$.

Theorem 11.6. Let $f$ be as above and let $m, r \geq 1$. There exist positive constants a depending only on $f$, and $C_{K}^{\prime}$ depending only on $K$, such that

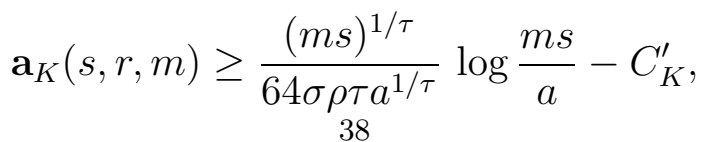


for all s sufficiently large.

Proof. By Corollary 6.5 there is $n_{r}$ such that

$$
Z_{n}(r) \leq a n^{\tau}, a=10 C\left(2 \beta^{-1}\right)^{1 / \gamma},
$$

when $n \geq n_{r}$. We fix $n_{0}=n_{0}(m, r) \geq n_{r}$ such that

$$
\left(m(n+1)^{2 \sigma-1}\right)^{-m / n} \geq 1 / 2,4 e^{4} r \leq n^{1 /(4 \rho)}, r_{n} \geq n^{1 /(2 \rho)},
$$

for $n \geq n_{0}$.

Let $s>a\left(2 n_{0}\right)^{\tau} / m$, and let $E$ be a subset of $\Delta_{r}$ with $|E| \geq s$. If

$$
n=\left[\left(\frac{m s}{a}\right)^{1 / \tau}\right]-1
$$

then $n>n_{0}$ and $m|E|>Z_{n}(r)$. Applying Theorem 10.4 as in the proof of Theorem 11.1, it follows that

$$
2 C_{K} \mathcal{A}_{K}^{2 \sigma}(E, m) \geq \exp \left(\frac{n}{4} \log \frac{r_{n}}{4 e^{4} r}\right) \geq \exp \left(\frac{n \log n}{16 \rho}\right) .
$$

Since

$$
n \log n \geq \frac{1}{2 \tau}\left(\frac{m s}{a}\right)^{1 / \tau} \log \frac{m s}{a}
$$

we obtain

$$
2 C_{K} \mathcal{A}_{K}^{2 \sigma}(E, m) \geq \exp \left(\frac{1}{32 \rho \tau}\left(\frac{m s}{a}\right)^{1 / \tau} \log \frac{m s}{a}\right),
$$

So

$$
\mathbf{a}_{K}(s, r, m) \geq \frac{(m s)^{1 / \tau}}{64 \sigma \rho \tau a^{1 / \tau}} \log \frac{m s}{a}-\frac{\log \left(2 C_{K}\right)}{2 \sigma} .
$$

We remark that versions of Theorem 11.4 and Corollary 11.5 can be stated for functions $f$ as in Theorem 11.6, by requiring that $\rho<\sigma / \tau$ and replacing the "liminf" in the conclusion by "lim".

\section{REFERENCES}

[B] A. Baker, Transcendental Number Theory, Cambridge University Press, 1975

[BBLT] L. Bos, A. Brudnyi, N. Levenberg, V. Totik, Tangential Markov inequalities on transcendental curves, Constr. Approx., 19 (2003), 339-354

[Br] A. Brudnyi, Local inequalities for plurisubharmonic functions, Ann. of Math. (2), 149 (1999), 511-533

[CP1] D. Coman, E. A. Poletsky, Bernstein-Walsh inequalities and the exponential curve in $\mathbb{C}^{2}$, Proc. Amer. Math. Soc., 131 (2003), 879-887

[CP2] D. Coman, E. A. Poletsky, Measures of transcendency for entire functions, Mich. Math. J., 51 (2003), 575-591 
[D] J. Dufresnoy, Sur les domaines couverts par les valeurs d'une fonction méromorphe ou algébroide, Ann. Sci. École Norm. Sup., 58 (1941), 179259

[FN] Ch. Fefferman, R. Narasimhan, On the polynomial-like behavior of certain algebraic functions, Ann. Inst. Fourier (Grenoble), 44 (1994), 1091-1179

[G] A. O. Gelfond, Algebraic and Transcendental Numbers, Dover, 1960

[GS] F. Gramain, F. J. Schnitzer, Ganze ganzwertige Funktionen: Historische Bemerkungen, Complex methods on partial differential equations, Math. Res., 53, 151-177, Akademie-Verlag, Berlin, 1989

[H] W. K. Hayman, Meromorphic functions, Oxford, 1964

[K] M. Klimek, Pluripotential theory, Oxford Univ. Press, New York, 1991

[La] S. Lang, Introduction to Transcendental Numbers, Addison-Wesley, 1966

[Le] B. Ya. Levin, Distribution of Zeros of Entire Functions, Transl. Math. Monographs, vol. 5, Amer. Math. Soc., Providence, RI, 1964

[M] K. Mahler, Lectures on Transcendental Numbers, Lect. Notes Math., 546, Springer, 1976

[RY] N. Roytwarf, Y. Yomdin, Bernstein classes, Ann. Inst. Fourier (Grenoble), 47 (1997), 825-858

[Sa] A. Sadullaev, An estimate for polynomials on analytic sets, Math. USSR Izvestiya, 20 (1983), 493-502

[Sc] T. Schneider, Ein Satz über ganzwertige Funktionen als Prinzip für Transzendenzbeweise, Math. Ann., 121 (1949), 131-140

[So] M. L. Sodin, Zeros and units of entire functions, Ukr. Math. J., 40 (1988), 91-95

[St] E. G. Strauss, On entire functions with algebraic derivatives at certain algebraic points, Ann. Math., 52, (1950), 188-198

[Ti1] R. Tijdeman, On the number of zeros of general exponential polynomials, Indag. Math., 33 (1971), 1-7

[Ti2] R. Tijdeman, On the algebraic independence of certain numbers, Indag. Math., 33 (1971), 146-162

[T] E. C. Titchmarsh, The Theory of the Riemann Zeta-Function, Oxford, 1986

[W] M. Waldschmidt, Pólya's theorem by Schneider's method, Acta Math. Acad. Sci. Hungar., 31 (1978), 21-25

Department of Mathematics, 215 Carnegie Hall, Syracuse UniverSITY, SyraCuse, NY 13244-1150, USA. E-MAIL: DCOMAN@SYR.EDU, EAPOLETS@SYR.EDU 